\title{
Corrupción, Estados débiles y racionalismo económico en Europa central y oriental
}

\section{Leslie Holmes*}

\section{Introducción}

Si bien la naturaleza misma de la corrupción determina que nadie puede saber a ciencia cierta de su existencia, existe una percepción generalizada de que se ha producido un incremento de la corrupción, prácticamente en todo tipo de sistemas políticoeconómicos desde los años ochenta ${ }^{1}$. Sin embargo, el problema parece ser mucho más grave en los Estados postcomunistas, más que en otro tipo de sistemas. Más aún, muchos consideran que el crecimiento dramático en la corrupción y, a menudo ligado a él, el crecimiento del crimen organizado, resultan ambos igual de impactantes en el caso de países situados más allá de la Europa Central y 0 riental (de ahora en adelante ECO). Esta cuestión es relevante.

Este trabajo se divide en tres partes principales. En la primera de ellas se proporciona un esquema general de la escala y naturaleza del problema. En segundo lugar paso a tomar en consideración sólo unas pocas de las múltiples razones que explican el aumento aparentemente dramático de la corrupción en los países de la ECO en los años noventa. Una de la propuestas controvertidas llevadas a cabo en esa sección es la consistente en afirmar que uno de los factores principales que viene a explicar la existencia de corrupción es el contexto internacional en el que surgió el post-comunismo. Se argumenta que la ideología neoliberal (racionalista económica), que ha venido a dominar una parte tan importante del mundo occidental, en las dos últimas décadas, ha tenido un impacto muy importante en los niveles de corrupción delos países EC 0 , aunque de un modo complejo. Si bien el neoliberalismo podría conducir a crecientes niveles de corrupción en los Estados occidentales, resulta, sin embargo, especialmente problemático cuando se combina con el legado comunista de los países de EC O : pese a ello, esta fórmula resulta aún mejor que la que resulta de la combinación del legado comunista y de la reforma parcial (incompleta). Seguidamente, se pasa a examinar hasta qué punto parece haber algún tipo relación entre la ideología del neoliberalismo y la debilidad del Estado post-comunista. La tercera sección se centra en los métodos que pueden adoptarse para combatir la corrupción. En las conclusiones que siguen a esas tres secciones principales se plantea hasta qué punto se está exagerando el problema de la corrupción en los países de ECO, y hasta qué punto viene a ser un mecanismo que permiteel funcionamiento de las construcciones estatales occidentales. Una vez argumentado que el problema es realmente serio, se viene a mantener que las implicaciones sociales y políticas de la corrupción resultan notablemente aún más preocupantes en los países postcomunistas que en los Estados occidentales.

\section{Corrupción en los países ECO}

Durante los años noventa en los países de ECO ha habido numerosos casos de corrupción oficial tanto reales, en el sentido de que han sido subsiguientemente probados, como alegados, aunque sin verificar en último término. Dichos casos van de 10 más alto de la jerarquía estatal hasta los estratos más locales, y a Io largo de todas las agencias estatales. De una u otra forma, la corrupción ha amenazado, o ha afectado de forma más negativa a las carreras políticas de los presidentes postcomunistas (tal es como el presidente de Polonia Aleksander Kwasniewski ${ }^{2}$; varios primeros ministros (incluyendo al primer ministro de Albania, Fatos N ano en 1991 3; al primer ministro de Polonia, Waldermar 
Pawlak en 1995; al primer ministro de Lituania, Adolfas Slezevicius a principios de 1996: al primer ministro de Estonia Tit Vahi en febrero de 1997; al primer ministro de Latvia, Andris Skele en julio de 1997; al dela República Checa, Vaclav Klaus, a finales de 1997; al de Georgia, Niko Lekishvili en julio de 1998; al de Lituania, Gediminas Vagnorius en abril de 1999 4), a numerosos oficiales superiores de privatización (incluida la privatización polaca del ministro Janusz Lewandowski en 1993; a la persona al mando de la privatización checa, Jaroslav Lizner, en $1994{ }^{5}$ y todo el comité de privatización en Hungría en octubre de $\left.19966^{6}\right)$; oficiales de policía (por ejemplo en Polonia a finales de los años noventa ${ }^{7}$, en Yugoslavia en $1998^{8}$ ), oficiales militares (como en Estonia a finales de $1995^{\circ}$, en Rusia a mediados de $1996{ }^{10}$, en Bulgaria en $1997^{11}$ en Rumanía a principios de $1998{ }^{12}$ en Eslovaquia a mediados de $19988^{13}$, y en Latvia, a finales de $1998{ }^{14}$ ) y muchos otros oficiales públicos. Incluso después de haber dejado de ocupar sus cargos, cabe seguir haciendo al egaciones de corrupción contra políticos anteriores y otros oficiales; en el momento en que escribimos este trabajo, (a mediados de 1999), el ejemplo más notable de esto fue toda la serie de acusaciones de desfalco vertidas contra el anterior primer ministro ucraniano, Pavlo Lazarenko ${ }^{15}$, que lo fue hasta junio de 1997, y también las denuncias formales de desfalco presentadas contra el anterior primer ministro de Bielorrusia, Mikhail Chyhir ${ }^{16}$.

Tal y como hemos hecho alusión al comienzo de este trabajo, la naturaleza misma de la corrupción hace que resulte imposible proporcionar datos fiables acerca de su escala y su incidencia en ningún país. A diferencia de lo que ocurre con la mayor parte de los otros crímenes, a menudo la corrupción carece de una svíctima» clara, que pudiera en su caso denunciar dicho crimen a las autoridades. En aquellos casos de corrupción en los que están involucrados al menos dos agentes ( por ejemplo, el que soborna y el sobornado), existe a menudo un interés mutuo en ocultar dicha actividad impropia al Estado. D esgraciadamente, esto sucede incluso en aquellos casos en los que una de las partes implicadas se ha mostrado inicial mente reticente a involucrarse en dicha actividad corrupta. Al ciudadano puede no gustarle tener que pagar un soborno al funcionario para que este último proporcione bienes o un determinado servicio al que el ciudadano crea que tiene derecho de por sí; pero una vez que se ha pagado el soborno, el funcionario en la mayor parte de los casos optará por no denunciar la actividad de meat-eating ${ }^{17}$ del empleado público por miedo a ser castigado por las autoridades. En aquellos países que se perciben como especialmente corruptos, puede incluso que los ciudadanos crean que existe un alto nivel de probabilidades de que el oficial de policía ante el quedenuncian el crimen resulte ser el mismo o ella misma corrupto, en cuyo caso el problema se vería acentuado. Resulta así relevante, por ejemplo, que en una encuesta relativamente reciente dirigida por el Centro de Estudios D emocráticos de Bulgaria, puso de manifiesto que el $57 \%$ de los encuestados defendía que era una «pérdida de tiempo» de nunciar los casos de corrupción, y que un porcentaje casi idéntico (56\%) créía que los oficiales de policía aceptaban de buena gana sobornos, mientras que el $63 \%$ créa que los jueces lo hacían ${ }^{18}$.

A la vista de estos resultados, resulta evidente por qué las estadísticas acerca de la corrupción deben tratarse con extrema precaución. Lo máximo que podemos obtener de las mismas re buscando en ellas es que nos muestran algún tipo de evidencia del mínimo alcance del problema. Más allá de eso, la información más útil, en cuanto a la valoración de las potenciales implicaciones sociales y políticas de la corrupción, son las percepciones públicas del problema. Si bien esto puede basarse en una importante sobreestimación de la escala del problema, son esas percepciones más que la realidad, las que importan en lo relativo a la legitimidad del Estado y a los problemas de transición. El resto de esta sección entra a considerar algunas de las estadísticas disponibles en materia de corrupción en los países de ECO, se guido de parte de los datos de encuestas y de grupos de investigación publicados. Esta visión general no puede ser sistemática por dos razones principales. La primera de ell las es una razón práctica de espacio, el simple resumen de las numerosas encuestas relacionadas con la corrupción llevadas a cabo en tantos países del ECO durante los años noventa requerirá dedicar muchas más páginas de las que aquí podemos destinar a esta cuestión. En segundo lugar, en la mayor parte de los casos, ni las estadísticas en materia de corrupción, ni los datos de los informes pueden establecerse de forma estándar a lo largo de las distintas formas de gobierno, de tal forma que no son posibles las comparaciones directas. Por tanto, lo que sigue va un poco al azar y de forma impresionista. M ientras que la gran mayoría de las estadísticas criminológicas hechas en todos los países post-comunistas son mucho más fáciles de obtener ahora de lo que lo era durante la era comunista, aquellos en materia de corrupción aún están bastante en retazos ${ }^{19}$. Rusia es uno de los países post-comunistas que sí proporciona estadísticas relativamente detalladas en materia de corrupción. Las estadísticas normalmente se presentan en tres categorías de corrupción entre funcionarios estatales y funcionarios públicos: desfalco, sobornos y abuso de cargo. Aunque el número de delitos recogidos se ha incrementado en cada una de las dos últimas categorías desde finales de 1991, el número de casos registrados de desfalco (que es sin comparaciones la categoría más amplia) se ha reducido en la actualidad. Sin embargo, el número de casos de desfalco registrados en los que etán implicados funcionarios se mantuvo en 35.460 en 1995 (en comparación con los 40.364 en 1991). Las cifras de 1995 de sobornos y abusos de cargo fueron respectivamente de 4.889 y 3.240 (en comparación con los 2.534 y 2.451 en 1991, o los incrementos en los porcentajes, de $93 \%$ y $32 \%$, respectivamente, en sólo cuatro años) ${ }^{20}$. Bulgaria representa un caso más típico que el de Rusia, en la medida en que proporciona escasas estadísticas específicas en materia de corrupción. Pero sí que sabemos que, entre 1991 y 
1995, sólo se recogieron por escrito 370 actos de soborno ${ }^{21}$ ( 10 que supone una media de 74 p.a..); cualquiera con algún tipo de conocimiento de la Bulgaria poscomunista apreciará que tales cifras parecen ser ridículamente bajas. Las cifras pueden haber crecido bajo el actual gobierno, con aproximadamente $100 \mathrm{em}$ pleados del Estado (especialmente agentes de aduana y funcionarios del Ministerio del Interior) por posible corrupción en noviembre de 199722.

Incluso en aquellos casos en los que un número relativamente alto de funcionarios es acusado de corrupción, los niveles de procesamiento y condena, al igual que ocurre en el 0 este, son a menudo sorprendentemente modestos ${ }^{23}$. Por ello, pese a los numerosos escándalos y alegaciones en materia de corrupción llevados a cabo en Polonia ${ }^{24}$, sólo cuatro funcionarios publicos fueron condenados por corrupción durante el periodo de siete años de 1990 a 1996, de acuerdo con el Ministerio de Justicia polaco ${ }^{25}$. D e forma similar, sólo 17 funcionarios fueron declarados culpables de estar involucrados en sobornos en Bulgaria en $1995{ }^{26}$, pese al hecho de que, menos de 18 meses después, se eligió un nuevo gobierno de coalición que había convertido su lucha contra el ampliamente extendido problema de la corrupción en uno de los temas esenciales de su campaña política (lo cual sugiere claramente que la corrupción era uno de los principales problemas, cualesquiera que fueran las estadísticas). Sólo dos jueces fue ron condenados por aceptar sobornos en Lituania, en el periodo de 1991-7, pese a la percepción popular existente de que la corrupción estaba ampliamente extendida en los organismos judiciales y en los organismos encargados de llevar a cabo la aplicación del Derecho ${ }^{27}$, Y sólo doce funcionarios (diez de ellos oficiales de policía) fueron acusados de aceptar sobornos en Cze quia en 1994 28. La mayor parte de estas cifras (posiblemente a diferencia de lo que ocurre con las cifras rusas) crasamente no se corresponden en absoluto con las percepciones públicas. Disponemos ya de pruebas a través de encuestas y de grupos de investigación para demostrar que la corrupción (al igual que el crimen organizado) es una de las principales preocupaciones en la mayor parte de los Estados poscomunistas. Una encuesta llevada a cabo en Rusia en mayo de 1992 puso de manifiesto que casi un 75\% de los encuestados 0 «estaban totalmente de acuerdo» (un 53.4\%) o si no «tendían a estar de acuerdo» (un 20.8\%), con la afirmación de que «la corrupción representa una amenaza muy seria a la sociedad». Los datos de timeseries dirigidos en Rusia entre 1994 y 1998 revelan que aunque sólo aproximadamente un cuarto de los encuestados (que subieron hasta el 30\% en enero de 1997 y el $32 \%$ en julio de 1998) consideraban que la corrupción era una de las amenazas más graves para ellos personalmente, con carácter más general, el crimen se percibía de forma consistente como la segunda, tercera o cuarta de las mayores preocupaciones, después de la inflación, y, en ocasiones, tras el impago de los salarios y las pensiones ${ }^{29}$. O tra encuesta realizada a lo largo del tiempo sobre qué era lo que los rusos más temían, reveló que el temor ante una «falta total de ley», «a criminalización de la sociedad» y la «corrupción de las estructuras de poder» (vlastnykh struktur) no venía a al canzar un nivel tan alto como el alcanzado en la primera encuesta citada; sin embargo, se situaban, respectivamente, en las posiciones 3.-, 4.0 y $7 . .-$ de las 43 posibles variables en 1996, y en las posiciones $4 .,-, 5 .-$ y 6.0 en 1998 . M ás aún, los porcentajes que optaban por estas tres variables en sus listados eran de nuevo de $67 \%$, 65\% y $53 \%$ respectivamenteen 1996 , en comparación con el 61\%, 61\% Y 54\% en 199830 .

Tambien podemos encontrar resultados similares a los hallados en Rusia en otros lugares; en multitud de países, las cifras son incluso más altas que las de Rusia. Una encuesta polaca de julio de 1991 reveló que un $71 \%$ de los encuestados considera que la corrupción es uno de los principales problemas, mientras que una encuesta de 1995 sugería que casi un 94\% de los polacos consideraban que la corrupción era un asunto importante o muy importante, por ejemplo ${ }^{31}$. Un informe más reciente del CBOS (abril de 1997) reveló que el porcentaje de polacos que consideraban que las máximas autoridades, incluidas el Parlamento, el gobierno y los ministerios, eran corruptas se había incrementado en un 50\% aproximadamente en los últimos años ${ }^{32}$. Las encuestas dirigidas en Eslovaquia durante los años noventa han indicado reiteradamente que la corrupción constituye uno de los tres problemas más serios para los ciudadanos ${ }^{33}$. Cuando en una encuesta de noviembre de 1997 se les pidió que nombraran únicamente un problema como el más serio en su país, un $41 \%$ de búlgaros identificó como tal a la corrupción entre funcionarios públicos; siguiendo los pasos de ésta en segundo lugar, como primera eleccion de un $24 \%$ de los encuestados eligieron 'Ios abusos a la propiedad privada' ${ }^{34}$. En julio de 1995 la encuesta realizada por el Banco M undial a 200 empleados lituanos reveló que un $54 \%$ de los que operaban en Vilnius y Kaunas había pagado sobornos a los empleados ${ }^{35}$. Una encuesta de 1997 hecha a 1.017 lituanos reveló que 'la criminalización de la sociedad', 'la corrupción en las estructuras de poder' y 'la falta total de leyes'ocupaban respectivamente las posiciones 6., a $7, \underline{a}$ y $3 . \underline{a} 36$, de entre los 45 posibles factores que los encuestados podían citar como aquellos que más les asustaban de su país, mientras que una encuesta similar, hecha a 450 ucranianos en 1998, situó estas variables (esta vez de entre 43 variables más que de entre 45), en la 6,7 y 3 posición, respectivamente. Finalmente, un informe del Banco M undial de 1998 en Latvia reveló que alrededor de un 60\% de las compañías y hogares entrevistados consideraban que la corrupción había aumentado en su país en los cuatro años anteriores, y que la mayor parte de la gente cré́a que esto se debía a una falta de compromiso para combatir la corrupción por parte de los gabinetes ministeriales ${ }^{37}$.

D esgraciadamente, no es posible probar, sobre la base de los resultados de los informes existentes o de las metodologías existentes, que la extendida preocupación sobre la corrupción en los países post-comunistas esté en modo alguno debilitando los legí- 
timos intentos llevados a cabo por parte de los gobiernos en estos países. Sin embargo, no parece infundado sugerir que las limitadas pruebas disponibles de Zazakhsthán y U zbekistán, que indican que muchos ciudadanos mantienen una postura ambivalente acerca de la democratización y de la reforma económica, precisamente porque asocian estos procesos con el incremento percibido en la corrupción y el crimen organizado ${ }^{38}$, puedan ser imitados en otros Estados post-comunistas donde se han dirigido encuestas similares. D os pruebas más pueden al egarse aquí para apoyar esta proposición.

En primer lugar, está el hecho de que tantísimos políticos a la cabeza en el mundo post-comunista se han centrado en la cuestión de combatir la corrupción al tratar de aumentar su popularidad (en especial durante las campañas electorales). Ejemplos de esto incluyen al presidente Constantinescu en Rumanía, Lukashenka en Belarus y Yeltsin en Rusia, y al Primer M inistro Kostov en Bulgaria. Hacen esto porque perciben que esta cuestión es una de las que más preocupan a nivel popular.

En segundo lugar, la reciente investigación basada en grupos de investigación y entrevistas, acerca de las actitudes existentes hacia la corrupción, dirigida por un equipo que tiene su base en la universidad de Glasgow, demuestra que existe preocupación extendida y bastante similar sobre este fenómeno en varios países de ECO. Dado que los cuatro países investigados (Bulgaria, la República Checa, Eslovaquia y U crania) representan una 'extensión' bastante amplia, en cuanto al nivel de desarrollo económico, la cultura política y las tradiciones religiosas, la proximidad al 0 este, etc., el hecho de que muchos hallazgos fueran esencialmente similares claramente sugiere que las actitudes públicas hacia la corrupción se han duplicado más o menos a lo largo de muchos de los países de la CEE. M ientras que un análisis detallado de estos hallazgos se escapa del ámbito de este artículo, resulta pertinente realizar algunas observaciones con carácter general.

Aunque los rankings relativos sobre quétipos de funcionarios públicos se percibían como los más corruptos, y cuáles los menos, variaban algo de un país a otro, el elemento más saliente es que todos los grupos de empleados públicos eran considerados corruptos por parte deal menos un 40\% de los ciudadanos en los cuatros países, y por más de la mitad de los ciudadanos en todos los países con la excepción de la República C heca ( y siendo U crania el país donde se dio la media de porcentajes más altos). 0 tro hallazgo está en el hecho de que, aunque fueron los checos y los eslovacos los que manifestaron una mayor preferencia por un Estado 'limpio' (por ejemplo, sin corrupción) que los ucranianos y búlgaros - a menudo al parecer porque estos últimos se percibían como tan sumamente disfuncionales que la corrupción se consideraba como un mal necesario para garantizar la realización de los resultados deseados- , seguía habiendo una mayoría tanto en Bulgaria como en U crania que preferiría un sistema libre de corrupción. Por último, es evidente que los ciudadanos en todos estos Estados son menos tolerantes con altos niveles de corrup- ción que con comportamientos incorrectos entre funcionarios locales; y también es evidente que la gran mayoría de los ciudadanos de los cuatro países consideraban los comportamientos impropios entre funcionarios del Estado más molestos que este tipo de comportamientos llevado a cabo entre empresarios ${ }^{39}$.

Junto con encuestas realizadas a ciudadanos en el marco de los propios Estados post-comunistas, existen numerosas encuestas de percepciones extranjeras. Por ejemplo, el informe del Banco M undial de julio de 1995, realizado en Lituania al que nos hemos referido con anterioridad, también se dirigió a 200 empresarios extranjeros que habían invertido en el país. De éstos, un 80\% alegaban que se les había pedido que pagaran sobornos, mientras que un $90 \%$ indicaba que la corrupción impedía invertir más en Lituania ${ }^{40}$. Pero, sin duda, el índice de la corrupción percibida citado con mayor frecuencia desde mediados de los años noventa ha sido el 'índice de percepción de corrupción' (al que a partir de ahora nos referiremos como CPI) producido y publicado por «Transparency International» (TI), junto con el Dr. Johann Graf Lambsdorff de la Universidad de Göttingen, anual mente desde 1995. El índice de 1998 abarca más que ninguno de los anteriores, incluyendo a 85 países de todo el mundo. De éstos, doce son Estados post-comunistas de la CEE. De acuerdo con el índice, se percibe que Estonia es el menos corrupto de los países de la CEE ( en la posición n- 26), mientras que Rusia es el más corrupto (en la posición 76); en orden ascendiente respecto del grado de corrupción percibida, los países que intervinieron son Hungría (que ocupa la 33 posición), la República Checa (la posición 37), Polonia (la posición 39), BieIorrusia y Eslovaquia (conjuntamente la posición 47), Rumania y Yugoslavia ( conjuntamente la posición 61), Bulgaria (la 66), U crania (69) y Latvia (71). Sin que sorprenda en absoluto, se percibe que en conjunto los países post-comunistas resultan ser más corruptos que los Estados occidentales, aunque menos corruptos que los países latinoamericanos o los Estados africanos sub-saharianos 41

Una última encuesta que merece mención es el estudio ' $\mathrm{N}$ aciones en Tránsito-1998' de 'Freedom H ouse'. Entre las múltiples fórmulas por medio de las cuales se clasifica a los países postcomunistas en este estudio, se encuentra la realizada en función de los niveles de corrupción. 'Freedom H ouse' sugiere cuatro categorías: de la A (niveles más bajos de corrupción) a la D (niveles más altos de corrupción). En la categoría D se encuentran Albania, Armenia, Szerbaijan, Kazakhstan, Kyrgyzstan, Rusia, Tajikistan, U crania y U zbekistán. Por tanto, con la excepción de Albania, todos los países con 'los más altos niveles de corrupción' son Estados pertenecientes con anterioridad a la Unión Soviética. El siguiente grupo con niveles altos incluye a varios Estados de Europa Central y del Sureste (junto a estos tres ex Estados de la Unión Sovietica), la lista incluye a Belarus, Bulgaria, C roatia, Georgia, M acedonia, M oldova, Rumania y Eslovaquia. En el grupo B están los tres Estados post-comunistas del Báltico (ej. Esto- 
nia, Latvia, Lituania) y la República C heca, mientras que el grupo menos corrupto (A) esta formado por Hungría, Polonia y Eslovenia. Para esos países esta lista no contiene muchas sorpresas. Sin embargo, merece la pena resaltar que 'Freedom House' da una posición a Latvia, muy diferente de la posición que le atribuye la TI ${ }^{42}$. Llegados a este punto de mi labor de investigación, la pura intuición sugiere que Latvia está más cerca de la clasificación dada por 'Freedom H ouse', que de la dada por TI, especialmente ya queTI considera a Latvia más corrupta que U crania.

\section{Las razones para la corrupción}

Existen muchísimas razones para la existencia de la corrupción en el mundo post-comunista. M ás que desarrollar todas ellas aquí - lo cual dada la limitación de espacio, requeriría que nos limitáramos a proporcionar una lista superficial de las mismasnos centraremos solamente en tres factores que son bien totalmente 0 en su mayor parte propios del post-comunismo.

El primero de ellos es el legado del comunismo. Varios analistas se han referido a 'la dependencia en el camino' - que en este contexto significa que un país determinado tiene una limitada gama de opciones entre las que elegir durante su fase de transición y que tiende a elegir unas más que otras, lo cual viene a su vez determinado por el contexto del que surge ${ }^{43}$ - para explicar la amplia gama de aspectos del comportamiento post-comunista. En contra de lo que al gunos analistas comparados parecen haber creído ${ }^{44}$, hubo una cantidad importante de corrupción en el mundo comunista ${ }^{45}$. Sin duda, la mayor parte de los políticos post-comunistas han defendido que es precisamente el legado del comunismo el que ha llevado a que existiera tanta corrupción en los países ECO en los noventa; el entonces presidente de Albania, Sali Berisha, hizo esta observación en diciembre de 1994, por ejemplo ${ }^{46}$. Cabe citar multitud de aspectos de este legado para explicar tal corrupción de 'la dependencia del camino'. Pero uno de los aspectos que cabe resaltar, ya que tantísimos ciudadanos se refieren a él cuando se les pide que sugieran explicaciones para la corrupción post-comunista, es el declive de la coerción. Aparentemente, multitud de ciudadanos creen que, como reacción directa a los métodos coercitivos 'totalitarios' que se aplicaron durante la era comunista, las sanciones contra los empleados públicos corruptos son ahora muy leves. En cualquier caso, la percepción que se tiene es que incluso estas débiles sanciones se aplican tan rara vez (algo que parece que los datos polacos citados más arriba respaldan ${ }^{47}$ ), que apenas les convence para no implicarse en la corrupción.

En segundo lugar unos de los aspectos que diferencia a los Estados post-comunistas de las sociedades en transición en otros lugares (como en Latinoamérica, el sur de Europa desde la mitad de los años setenta, o Sudáfrica) es el hecho de que han estado sujetosa múltiplesy simultáneastransiciones desdela caída del poder comunista en 1989-91. M ientras que los demás Estados en transición tuvieron que llevar a cabo revoluciones políticas, los Estados post-comunistas tuvieron, además, que introducir cambios fundamentales en el orden económico, legal, ideológico y social. M uchos de ellos tuvieron, además, que redefinir sus fronteras y sus identidades, y realinearse en los bloques internacionales militares y comerciales. El ámbito tan completo de cambio que se trató de llevar a cabo es una de las razones más importantes por las que la mayor parte de los Estados post-comunistas han sufrido un grave retraso legislativo en la última década. Este retraso ha supuesto que básicamente no han existido leyes, o si han existido han sido muy vagas y contradictorias. Esta situación es muy propicia tanto para la corrupción como para el crimen organizado 48 .

El último punto en esta sección se refiere al contexto internacional en el que surgió el post-comunismo. Se acepta con carácter general queel surgimiento del post-comunismo coincidió con una recesión en el 0 este, y que el amplio número de Estados en los que el poder comunista fue rápidamente derribado, de forma más o menos simultánea, sólo vino a añadir más presión general al capital extranjero. Esto supuso que había menos inversión disponible para países post-comunistas individualizados, de la que hubiera habido si la transición del comunismo a la democracia se hubiera dado entonces en sólo tres países. Pero éste no es el único aspecto de la situación económica internacional que ayuda a explicar la corrupción en el mundo post-comunista durante los años noventa. 0 tro factor, que típicamente se pasa por alto, es que el nacimiento de post-comunismo coincidió con el creciente impe rio de la ideología neoliberal (racionalista económica) en el 0 este. Esto requiere un mayor desarrollo.

La extensión del racionalismo económico en el 0 este se asocia con carácter general con la llegada al poder de M argaret T hatcher en el Reino Unido (1979) y de Ronald Reagan en EE.UU. (electo en 1980); sus principales teóricos economistas son los miembros del Ilamado «C hicago School», delos queM ilton Friedman es el más conocido. Esta versión del capitalismo es una versión más pura y una forma mayor de estratificación social que el keynesianismo o la aproximación económica de la democracia social. D efiende minimizar el papel del Estado en la economía, y animar a la sociedad - en teoría a la sociedad civil- a asumir muchas de las responsabilidades que habían sido aceptadas por el Estado en muchos países de Europa 0 ccidental, en las recientes décadas. Una de las fórmulas a través de las cuales ocurre esto es a través de la privatización de los servicios y de las industrias que en su momento llevó el Estado. Incluso en aquellos casos en los que el Estado sigue aceptando responsabilidad por los servicios prestados, el método utilizado por el mismo para suministrarlos difiere deforma sustancial delas prácticas anteriores. Setiendea huir de los monopolios dirigidos por el Estado hacia la competencia, 
el marketing, y las relaciones outsourcing y contractuales, la noción del 'usuario-paga', y la maximización del coste-efectividad.

Algunosanalistas han argumentado queestecambio en el papel del Estado conduce necesariamente a una reducción en el nivel de corrupción, simplemente porque, al ser el Estado más peque ño (ej.: tiene menos empleados públicos), existen menos oportunidades para que haya corrupción ${ }^{49}$. Resumiendo, esa argumentación dice que ceteris paribus cuantos menos empleados públicos tenga el Estado, menor será la corrupción. A primera vista, parece convencer en su simplicidad.

Pero los argumentos simples no son siempre los más convincentes a la hora de analizar las complejidades de la sociedad humana, y existen poderosos argumentos en contra de éste.

Por ejemplo, el proceso mismo de privatización - que en ultimo término es implementado por el Estado- da nuevas oportunidades a los empleados públicos. Pueden (y la investigación empírica demuestra que a menudo lo hacen) exigir o pedir sobornos o compensaciones en secreto de los agentes privados interesados en adquirir un negocio que pertenece en ese momento al Estado, de una forma en la que no pueden cuando no existe un proceso de privatización. D ebemos admitir que, en el caso especial de la privatización, existe un dinamismo respecto del proceso que determina que, una vez que el proceso se ha completado, también desaparecen las oportunidades. En el mundo real, la situación puede no ser tan clara como está, ya que el Estado puede seguidamente optar por la renacionalización, que a su vez abre la vía a posteriores reprivatizaciones. Pero no ensuciemos las aguas con escenarios tan sumamente hipotéticos. En cambio, merece la pena centrarse en otras dimensiones del racionalismo económico que puede tener implicaciones para la corrupción.

La mayor parte de los neoliberales están a favor de gestionar los servicios externamente, pero a menudo no admiten las potenciales implicaciones negativas que esto puede tener para la corrupción. Siendo los demás aspectos iguales, existen menos oportunidades para que los empleados públicos soliciten o exijan sobornos o compensaciones en secreto en aquellos casos en los que un municipio gestiona su propio servicio (monopolístico) de recogida de basura que en aquellos otros en los que los agentes privados pujan de forma colectiva en una subasta de forma competitiva por el contrato que lleve a cabo estas tareas. Dado que uno de los principios esenciales del argumento neoliberalista es quela gestión externa de los servicios resulta más efectiva en cuanto a los costes precisamente porque implica competitividad, se debe fijar regularmente el itinerario de dicho delicado proceso si se quiere conseguir el efecto deseado. Esto significa que existen continuamente posibilidades de corrupción entre estos empleados públicos involucrados en la adjudicación de contratos.

D esgraciadamente, los movimientos del Estado hacia una gestión externa de los servicios y hacia una comercialización de las funciones que en su momento realizaba a menudo conduce a un mayor secretismo (menos transparencia). Las leyes de confianza en el comercio normalmente son de aplicación en aquellos casos en los que, si el Estado siguiera aún realizando tareas que ahora tiene transferidas al sector privado, las leyes de la libertad de información hubieran permitido a los ciudadanos investigar aque Ilas acciones y transacciones que son cuestionables. Esa reducción de la transparencia tiene muchas posibilidades de llevar a la corrupción.

0 tro aspecto del racionalismo económico que puede ayudar a explicar el aparente incremento de la corrupción en los Estados occidentales en los últimos años es la reducida lealtad que produce. En el pasado, una persona podía optar por una carrera profesional bien en el sector privado o en el sector público suponiendo que las condiciones fuesen diferentes. En términos claros, un puesto en este último resultaría menos arriesgado (ej.: habría una mayor seguridad en el puesto de trabajo, un camino profesional más fiable, y un mejor sistema de pensiones), pero también sería potencialmente menos lucrativa que una posición en el sector privado. Pero con la adopción de muchos aspectos del modus ope randi del sector empresarial privado por parte de las agencias estatales, incluida una seguridad mucho menor del puesto de trabajo, muchos empleados consideran que su empleador no les es tan fiel como antes, lo que genera en respuesta a eso una reducción de la propia lealtad de los empleados hacia el empresario. A menudo este problema se mezcla con el hecho de que los salarios y los complementos salariales en el sector estatal no son equiparables a los del sector empresarial privado. Por tanto, los trabajadores al servicio del Estado pueden tener la impresión de que están perdiendo el doble, ya que aunque están sujetos a las mismas inseguridades, al mismo estrés y a la misma competitividad que el sector privado, no tienen normalmente acceso a los paquetes altamente lucrativos a los que tienen acceso los trabajadores con éxito del sector privado. El problema se complica aún más, si los empleados públicos consideran que los elementos criminales tienen un papel esencial a la hora de beneficiarse del sector privado y, además, hacerlo sin que les cojan. Todo esto puede conducir a resentimientos, y aumenta las probabilidades de que los empleados públicos se involucren en la corrupción.

Finalmente, e íntimamente ligado a este último punto, la creciente interacción entre el Estado y el sector privado de la economía, típica de los neo-liberalistas occidentales, tiende a difuminar las fronteras entre ambos ${ }^{50}$. ¿Cómo se relacionan estos aspectos con los Estados post-comunistas de la ECO?

Tal y como ocurrió en los países latinoamericanos en los años ochenta, varios países post-comunistas sehan visto explícitamente influenciados por las ideas neo-liberales. A principios de los años noventa, los ejemplos más claros de ello fueron Polonia y Rusia, en los que Leszek Balcerowicz y Egor Gaidar, respectivamente, reconocieron la influencia de los defensores occidentales del racionalismo económico radical, tales como el economista de $\mathrm{H}$ arvard Jeffrey Sachs. En Czequia, Václav Klaus a menudo cita a 
M argaret Thatcher y a su modelo y heroína en lo que se refiere a la gestión económica. A menudo se cita por parte de los neoliberales a Estonia, con su política de impuesto de renta con un índice bajo y estable, como modelo bueno (eficiente) de gobierno post-comunista. M ás recientemente, los gobiernos que llegaron al poder en Rumania en noviembre de 1996 y Bulgaria en abril de 1997 dejaron claro su compromiso con principios básicamente neoliberales. M ientras que otros Estados del ECO se han mostrado en la mayor parte de los casos menos abiertos con respecto a su compromiso con el racionalismo económico, no cabe apenas ninguna duda de que se ven afectados por la prevaleciente ideología del 0 este; esto resulta aún más obvio cuando comparan sus propias formas económicas de actuación con las de varios países del ECO que abiertamente abrazaron los principios del racionalismo económico con anterioridad ${ }^{51}$.

D esgraciadamente, si bien las políticas neoliberales pueden haber ayudado a algunos países post-comunistas a recuperarse económicamente más deprisa de lo que lo hubieran hecho si no, también han contribuido al problema de la corrupción. Q uizá irónicamente, en la práctica al gunos aspectos del neoliberalismo incluso se superponen y se unen a los legados comunistas. De esta forma, la mención hecha anteriormente respecto a «la existencia de barreras difuminadas» tiene fuertes resonancias en la experiencia comunista, ya que no sólo resultaba difícil de determinar la línea de demarcación entre el Estado y la economía, sino que, además, la confusa relación entre 'el partido' y Estado aumentaba la poca claridad entre las fronteras en el sistema en todo su conjunto.

Si con carácter general el efecto de las políticas del racionalismo económico es el de aumentar la corrupción, el efecto probable de estas políticas en los países del ECO resulta más complicado. A un nivel, sin duda, añade nuevas dimensiones al problema. Por ejemplo, el problema occidental de la corrupción consistente en «compensaciones secretas» durante la fase de privatización, se entremezcla en los países del ECO, no debido únicamente a la escala y el ritmo de la privatización, sino también porque los empleados públicos a menudo han vendido activos del Estado a sí mismos, a precios muy por debajo de lo que el mercado hubiera fijado. Esto desacredita el proceso de privatización y priva a los Estados empobrecidos y luchando por mejorar su situación de beneficios.

0 tra de las razones principales por las que el neo-liberalismo ejerce una influencia negativa se debe al vacío ideológico entre el viejo sistema y el nuevo, que es mucho más amplio de lo que lo hubiera sido si los Estados post-comunistas hubieran tratado de adaptar el entonces llamado modelo nórdico o de la zona del Rin (ej.: un modelo más social demócrata). En este último caso, los empleados públicos se hubieran sentido menos dislocados, confundidos o inseguros y básicamente menos alineados, lo que a su vez hubiera hecho menos probable que tendieran a un comportamiento antisocial y antisistema.
Defender este último argumento no supone sugerir que los Estados del ECO deberían haber adoptado una versión del modelo nórdico de la zona del Rin. Lamentablemente, ésta no era una posibilidad realista, ni siquiera si los políticos de los Estados del ECO hubieran intentado conseguir esta opción. Este modelo social democrático sólo habría tenido éxito en países altamente desarrollados y ricos - y no cabría definir aśí a ninguno de los países de la ECO al principio de los años noventa (ni siquiera incluso a finales de los años $90{ }^{52}$ ) - . M ás aún, incluso los países escandinavos y germánicos que en su momento habían adoptado tal modelo se han ido separando a diferentes niveles de él en los últimos años y se han ido aproximando al modelo transatlántico, más despiadado e individualista. La llegada al poder de partidos de centro-izquierda en casi todos los países de la U nión Europea a mediados-finales de los años noventa, además de las referencias hechas por varios líderes occidentales al 'Tercer Camino' o al 'N uevo Centro', podría sugerir una vuelta al comienzo del siguiente milenio a algo similar al anterior modelo nórdico en al menos algunos de estos países (aunque uno de los teóricos a la cabeza de la nueva versión del Tercer Camino ha desechado explícitamente la posibilidad de esto en el contexto político competitivo de una economía globalizada ${ }^{53}$ ). Incluso si esto fuera a ocurrir, pasará mucho tiempo hasta que ninguno de los Estados del ECO tengan una economía suficientemente fuerte para hacer que ésta sea una opción factible ${ }^{54}$.

Por tanto, si bien un contexto internacional con una ideología neo-liberal dominante podría ayudar a explicar la existencia de niveles superiores de corrupción en los Estados post-comunistas, en último término no tiene sentido sugerir que estos países deberían haber tratado de seguir otra forma de desarrollo capitalista más 'considerada'. Simplemente esto no era una opción existente. Del mismo modo, simplemente por el hecho de que se utilice aquí la tesis del 'mayor vacío' ( por ejemplo, entre dos ideologías y dos sistemas) para proporcionar una explicación en parte del aumento de la corrupción en el mundo post-comunista, esto no debería interpretarse en el sentido de que existía una alternativa mejor; de hecho, se argumenta que el legado del comunismo determinaba que, en cualquier caso, iba a haber corrupción en estas sociedades, y que es mejor tener corrupción en una sociedad y en una economía que está progresando que en una sociedad estancada. Este punto conduce a los enigmas de Estonia y Polonia, dos de los países con más éxito de los Estados post-comunistas, y dos que parecerían debilitar la hipótesis aquí planteada. Los lectores observadores pueden haberse percatado de la aparente contradicción existente en el hecho de que Estonia ha sido citada tanto como el primer ejemplo de un país que ha adoptado las políticas racionalistas económicas radicales, y a la vez como el menos corrupto de los Estados postcomunistas del ECO conforme al ranking del IT (International Transparency) de 1998. Si bien esto es en cierta forma un enigma, se deberían tener en cuenta las siguientes cuestiones. 
En primer lugar, las repúblicas bálticas normalmente eran consideradas como las menos corruptas de entre las anteriores unidades federales soviéticas. Si tenemos en cuenta que los Estados del norte de Europa (Dinamarca, Finlandia, Suecia y N oruega) se perciben como los menos corruptos del mundo, entonces la posición de Estonia en la tabla causa menos impresión, de he cho, tiene un aspecto bastante bueno en relación con los demás Estados post-comunistas.

En segundo lugar, existen pruebas de que los medios de comunicación de Estonia no han denunciado la corrupción tanto como deberían haberlo hecho, al parecer porque el público de Estonia no reacciona frente a las denuncias de corrupción tanto como lo hacen los ciudadanos de otros países ${ }^{55}$. Dado que el índice del IT se basa principalmente en percepciones de los empresarios extranjeros, y asumiendo que unos pocos de éstos lean los periódicos de Estonia, inicialmente parecería que esta explicación es errónea o irrelevante. Sin embargo, las percepciones que tienen los extranjeros respecto del nivel de corrupción en un país determinado a menudo se forman, en parte, a raíz de conversaciones y otras formas de interacción con la gente que viveen ese país. D e este modo, si los propios ciudadanos de Estonia no consideran que la corrupción sea un problema esencial, hasta cierto punto como respuesta a los niveles comparativamente bajos de denuncia de este fenómeno llevadas a cabo por parte de los medios de corrupción, resulta aún menos sorprendente que los extranjeros tampoco lo hagan.

En tercer lugar, mencionamos con anterioridad que el nivel de corrupción podría incrementarse como resultado de un sentimiento de resentimiento por el hecho de que los elementos criminales estén beneficiándose de los nuevos arreglos económicos. No parece apenas necesario resaltar que ésta es una cuestión muy pertinente en muchos de los países del ECO. La influencia de elementos criminales externos rusos parece ser menor en el caso de Estonia que en el caso de sus países bálticos vecinos; pero sigue siendo un factor importante. Esto, unido al estado comparado de la economía y de las previsiones del bienestar social relacionadas con el legado comunista de estos países, nos ayuda a explicar por qué la corrupción en Estonia es mucho más grave que en los países nórdicos vecinos.

Por último, el enigma de Estonia sugiere que las ramificaciones del legado comunista constituyen un factor que explica de forma mucho más poderosa la influencia de las políticas económicas neo-liberales. Esta interpretación también resultaría compatible con el hecho de que Latvia, que ha llevado a cabo una reforma económica mucho menos radical que Estonia, pero que es también un Estado post-soviético (y no solo post-comunista), puntúe tan bajo en el índice IT 56.

Polonia no constituye un enigma tan aparente como Estonia, pero sí ha adoptado políticas neo-liberales en algunas áreas. Si bien su puntuación en el índice IT no es tan buena como la de
Estonia, sin embargo, puntúa bastante mejor que la mayor parte de los países post-comunistas, y es uno de los tres países en la lista de Estados post-comunistas 'menos corruptos' de «Freedom H ouses. De nuevo, determinados aspectos del desarrollo polaco en los últimos tiempos podrían ayudarnos a dar una explicación a esto.

Q uizá, la cuestión más importante sea precisamente el hecho de que su economía se haya desarrollado más que la de ninguna otra región. El rápido cambio de Polonia después de la caída del poder comunista no debe atribuirse únicamente a las políticas radicales de Balcerowicz, incluso si éstas fueron uno de los factores más relevantes. También ayudó el hecho de que se considerara a Polonia por parte de muchos en 0 ccidente como el país que tuvo un papel más importante en el ECO, acabando con el poder comunista en la región, en su mayor parte por medio de la Solidaridad; cuando el anterior líder de ésta y entonces presidente de Polonia, Lech Walesa, mandó una descripción de la deuda polaca a las instituciones occidentales, se le escuchó casi con mayor simpatía de lo que se habría escuchado a nadie en la región ${ }^{57}$. Eso hizo que Polonia tuviera un buen comienzo en su transformación económica que al menos tuvo dos implicaciones para la corrupción. En primer lugar, los empleados polacos no se volvieron tan desesperados como lo hicieron sus iguales en otras partes de la región, donde la economía no parecía mejorar mucho, si es que mejoraba algo. En segundo lugar, los polacos pudieron enorgullecerse con su país más de lo que lo hacían la mayor parte de las gentes del ECO, y estaban menos alienados que muchos de estos otros grupos. D esgraciadamente, estas dos explicaciones dadas son y pueden ser sólo especulativas. M ás aún, ninguna de ellas debería llevarse demasiado lejos. Polonia tuvo su dosis correspondiente tanto de corrupción como de decepción pública en los años noventa; todo lo que persigo aquí es sugerir motivos por los que los niveles de corrupción de Polonia parecen ser algo menores que los de otros países, y porque esto no viene a refutar per se el argumento neoliberalista expuesto con anterioridad. H emos llegado al punto en el que cabe examinar la relación entre el racionalismo económico y los Estados débiles.

A menudo se ha dicho que los Estados en transición son típicamente Estados débiles. Existen muchas razones para tal debilidad. U na de ellas es el nivel tan completo de cuestiones que se les ha exigido hacer - la múltiple transición simultánea a la que nos hemos referido con anterioridad-, lo que ha determinado que todos ellos hayan estado sobrecargados, y, por tanto, hayan sido menos efectivos de lo que hubieran podido serlo en circunstancias más estables.

Pero las implicaciones de esa sobrecarga de la transición múltiple en los países del ECO, no son los únicos aspectos relacionados con el legado comunista que explican la aparente debilidad de los Estados post-comunistas. 0 tro de estos motivos ya ha sido mencionado en otro contexto. Este motivo se encuentra en el hecho de que muchos políticos (al igual que muchos ciudada- 
nos) quisieran distanciarse lo máximo posible del autoritarismo de la era comunista. Relacionado con esto estaba el hecho de que a menudo quisieran minimizar el uso de burócratas de ese periodo; a la vez que encontraron que tendrían que confiar más de lo que hubieran preferido en gentes que habían sido empleados públicos durante la era comunista, estos últimos a menudo apreciaban que tendrían que cambiar sus actitudes y su estilo para sobrevivir, y no digamos para prosperar, en el marco de los nue vos arreglos llevados a cabo. El hecho de que hubiera «perros viej0s» tratando de enseñarse a sí mismos - o tratando de ser enseñados- los nuevos trucos puede provocar confusión, lo cual a su vez puede hacer que una organización funcione de forma menos efectiva. En aquellos casos en los que hay multitud de nuevos burócratas, puede pasar tiempo hasta que esas personas desarrollen confianza y experiencia en sus nuevos puestos, aunque la experiencia depende hasta cierto punto del tiempo. Todo esto nos ayuda a explicar la debilidad del Estado.

0 tra de las explicaciones significativas de la debilidad de los Estados post-comunistas se refiere a sus estructuras socio-económicas. Entre las muchas razones por las que se produjo un cambio relativamente pacífico del poder comunista al poder post-comunista en la mayor parte de los Estados del ECO está en el hecho de que las viejas elites políticas sabían con anterioridad los cambios que se avecinaban para sus sistemas económicos, y en último término para sussistemas políticos, y no habían hecho apenas nada para resistirse frente a la presión para el cambio. Incluso, lejos de resistirse a dicho cambio, muchos de ellos buscaron asegurarse un sitio en primera fila de esos nuevos ajustes, y en algunos casos incluso animaron la transformación. Muchos anticiparon que, en los nuevos sistemas, el poder económico sería en último término más importante que el poder político, en marcado contraste con la configuración previa. Éstas son las personas que han conducido hacia y se han beneficiado de la llamada 'privatización nomenklatura'. Ante la casi total ausencia de una burguesía natural del lugar, en cierta forma, este grupo se convirtió en un componente esencial de la nueva burguesía sustituta. Sin embargo, claramente no ha estado entre los intereses del grupo el crear un Estado de D erecho. Esto requiere de una mayor explicación.

En uno de los mejores artículos existentes hasta la fecha sobre el post-comunismo, el científico político de Harvard, Joel H ELLM AN ha argumentado de forma persuasiva que los ganadores del inicial post-comunismo, incluyendo a aquellos que se han beneficiado de la privatización, sólo han perseguido una reforma económica limitada. Básicamente, han querido la reforma suficiente que les permitiera beneficiarse de una privatización rápida pero a menudo muy mal dirigida; una vez que esto ya ha sucedido, han tratado de bloquear cualquier reforma posterior, ya que ven que sus puestos privilegiados están en peligro si se siguen produciendo cambios hacia una regularidad, transparencia y un Estado de D erecho. De acuerdo con H elLm AN, han perseguido, por tanto, lo que él Ilama 'el equilibrio de una reforma parcial'.
Como resultado, mientras que los ganadores han adquirido un primer beneficio del proceso de reforma, también han desarrollado un beneficio en las propias distorsiones que impiden que se produzcan ganancias en la eficiencia de un mercado que funciona completamente ${ }^{58}$.

Partiendo de la base de que este argumento es correcto, necesitamos redefinir, y de hecho modificar de forma sustancial nuestro análisis del impacto del neo-liberalismo en los Estados postcomunistas. Aunque cabe mantener aún muchas de las críticas hechas a la postura económico-racional ista, queda claro, a la luz de lo planteado por H ELLM AN, que los problemas de la transición post-comunista se han visto exacerbados por la adopción de forma incompleta del modelo neo-liberal. Expresándolo sin tapujos, los ciudadanos de los países de ECO han obtenido lo peor de los dos mundos. Por un lado, el legado con múltiples facetas de la era comunista está afectando al funcionamiento del Estado, aunque este legado sea más tangible en al gunos países que en otros. Por otro lado, los aspectos negativos del neo-liberalismo se están manifestando, mientras que las potenciales ventajas de esta ideología - una notable mayor eficiencia, precios más bajos, mayores opciones, más competencia, y menos poder para el Estadoa menudo son apenas perceptibles (de nuevo, el grado en el que lo son varía de un país a otro).

Se sostiene aquí que el análisis llevado a cabo más arriba, que se centra en el hueco existente entre la teoría y la práctica del neo-liberalismo y la inusual estructura de las sociedades postcomunistas, ayuda a explicar el aparente incremento de la corrupción en 0 ccidente desde los años ochenta - al igual que explica por qué la situación es incluso peor en los Estados postcomunistas, a la vez que existe una conexión entre la corrupción en los países del ECO y Europa 0 ccidental ${ }^{59}$. M uchos consejeros económicos occidentales no sólo no entendían las particularidades institucionales y culturales y las limitaciones de los países del ECO a los que trataban de influenciar, sino que también se les pasó por alto la cuestión importante relativa a la naturaleza clasista de las estructuras sociales.

\section{Control y pronóstico}

Existen numerosas formas de hacer frente a la corrupción. La mayoría de los métodos más apropiados y más conocidos no son propios de los Estados post-comunistas, y, por tanto, sólo los enumeraremos y desarrollaremos aquí brevemente ${ }^{60}$. Seguidamente, hay otra sección que se centra en analizar el dinamismo tanto del post-comunismo como de la corrupción, lo que nos permitirá después realizar una serie de hipótesis sobre los probables futuros escenarios de los países del ECO. 
Q uizá, el método más obvio utilizado para combatir la corrupción sea utilizar el peso de la ley. Llevar esto a cabo, especialmente frente a empleados superiores contra quienes hayan pre sentado cargos de corrupción, constituiría un indicador concreto de la consolidación del Estado de D erecho en los Estados del ECO ${ }^{61}$. En la actualidad, el número tan bajo de condenas por corrupción, comparado con el número de denuncias o alegaciones hechas, sugiere que queda aún mucho camino por recorrer. M ás aún, incluso en aquellos casos relativamente escasos en los que se demuestra que empleados públicos son culpables de corrupción, sus castigos son típicamente muy leves ${ }^{62}$. D e esta forma, de las 70 condenas dictadas en 1996 contra empleados polacos considerados culpables de aceptar sobornos, las condenas más graves han sido de dos años de prisión ${ }^{63}$. Sin duda, cabría argumentar que si los empleados llevan a cabo un crudo análisis coste-beneficio, para muchos de ellos resulta económicamente racional involucrarse en la corrupción incluso si después les pillan y son declarados culpables. Esto resulta muy problemático si los gobiernos son serios a la hora de combatir la corrupción.

Un segundo método relacionado con el anterior es el de afianzar los cuerpos responsables existentes para combatir la corrupción y los crímenes relacionados con ésta y/0 establecer nuevos cuerpos. Un ejemplo del primero fue el anuncio en 1996 de que el número de tropas ligado al M inisterio del Interior ruso había de incrementarse en 10.000 , especialmente para hacer la fuerza más eficiente a la hora de hacer frente al crimen organizado y a la economía negra. Entre los numerosos ejemplos del segundo que cabe citar está el establecimiento en julio de 1991 en Polonia de una fuerza especial de policía para combatir los crímenes económicos y la corrupción, la fundación de un grupo militar especializado en crimen, que se anunció por parte del nuevo primer ministro polaco O lesky en marzo de 1995; el establecimiento en 1996 de un grupo militar ligado al gobierno húngaro y cuya misión era combatir la corrupción, y el establecimiento en septiembre de 1997 de un Consejo para la Prevención de la C orrupción en Latvia. Además de establecer más agencias generales, cabe establecer a su vez cuerpos especializados que tengan como objetivo combatir la corrupción en determinados órganos estatales. Ejemplos de ello incluyen el anuncio ruso llevado a cabo en noviembre de 1995 del establecimiento de un nuevo departamento en el Ministerio del Interior fundado específicamente para investigar la corrupción dentro del propio M inisterio; y el Inspectorado General de Aduana que se inició en Polonia en 1997, como parte de un impulso para limpiar los servicios de aduana polacos ${ }^{64}$.

En tercer lugar, se puede mejorar la legislación. Esto se puede conseguir eliminando las lagunas ( 0 al menos reduciéndolas!), e introduciendo nuevas leyes para hacer frente a problemas que bien no existían o no eran percibidos como tales oficialmente durante la era comunista. Uno de los tipos de legislación que cabe introducir $-y$ de hecho así se ha hecho en varios países post-comunistas- en los noventa son las leyes de conflicto de intereses; los ejemplos incluyen a Latvia en 1996 y Polonia en junio de 1997. Durante la era comunista, normalmente no era posible que los empleados públicos fueran directores de empresas o que tuvieran acciones de compañías privadas; con los movimientos hacia la privatización y la marketización en el mundo post-comunista, esto no sólo resulta meramente posible, sino que ocurre a menudo, que tanto los empleados públicos electos como nombrados por el Estado se convierten en accionistas y miembros de los consejos de administración de las compañías. Como ocurre en 0 ccidente, la mayor parte de lo que es legal y lo que no lo es es bastante difuso hasta que se adopte una legislación que pueda trazar límites razonablemente claros ${ }^{65}$.

D esgraciadamente, ha habido varios casos en los años noventa en los que los políticos de los países del ECO han rechazado los intentos de limitar sus privilegios actuales o potenciales por medio de leyes de conflicto de intereses. Por ejemplo, en 1992, tras una importante crisis bancaria que reveló que muchos parlamentarios eran miembros de los consejos de compañías, hubo un intento de aprobar tal tipo de ley en Hungría; pero fue rechazada en el Parlamento en abril de ese año ${ }^{66}$. M ás aún, continúan fracasando los intentos por aumentar la transparencia respecto de los ingresos de los empleados en algunos Estados del ECO. En marzo de 1999, el Parlamento de Latvia votó en contra de una propuesta para conceder a los ciudadanos el derecho a acceder a una información más detallada acerca de los ingresos de los empleados tanto a nivel central como local ${ }^{67}$.

O tra área madura para el refinamiento legislativo es la de la financiación de los partidos políticos. La forma más familiar y obvia para enfrentarse a este problema es especificando las condiciones para la concesión de fondos. Estas condiciones pueden incluir detalles sobre quien puede otorgar fondos a los partidos (ej.: ¿debería la concesión de fondos ser exclusivamente del gobierno?, y si es así, ¿conforme a qué criterios? ¿Se debería permitir a los negocios conceder fondos a los partidos?, y si es así, ¿hasta qué nivel?); también pueden incluir detalles sobre si las donaciones hechas a partidos debieran declararse públicamente; y si ello fuera así, iesto afectaría a todas las donaciones o sólo a las contribuciones realizadas por exceso de una cantidad especifica$d a^{68}$ ? Pero, en especial en el contexto de la existencia de fondos limitados disponibles en los países post-comunistas (aunque yo defendería que esta aproximación también es recomendable incluso también en los países occidentales relativamente ricos), quizá tendría más sentido pensar lateralmente, y perseguir limitar el posible gasto de los partidos, más que tratar de supervisar las fuentes de ingresos. En esta conexión, resulta positivo que la Re pública Checa redujera las posibilidades de propaganda política en la campaña preelectoral en 1998, por ejemplo, prohibiendo el uso de pancartas. Siendo los demás aspectos iguales, en aquellos casos en los que se reducen los gastos, también se reduce la demanda de recursos extra (y a menudo extra-legales). 
Existen, sin embargo, potenciales inconvenientes si restringimos la legislación de los Estados que tratan a la vez de acercarse más a una base de Estado de D erecho. El más obvio es que algunas propuestas legislativas pueden potencialmente vulnerar la intimidad de los ciudadanos, y, por tanto, han sido criticadas por los ciudadanos y políticos preocupados por los derechos civiles. Un buen ejemplo de ello fue el intento en 1993-4 de conceder a la policía polaca la posibilidad de intervenir los teléfonos y montar operaciones puntuales en los casos de crímenes económicos y de corrupción ${ }^{69}$. Pero este caso también demuestra que al menos al gunas de las preocupaciones de los defensores de los derechos civiles pueden afrontarse en una forma que represente un compromiso razonable entre los derechos de los ciudadanos y la necesidad de combatir la corrupción; la ley que permitió que la policía se involucrara en estas dos actividades requería que los policías que quisieran escuchar esas conversaciones ajenas o que quisieran 'atrapar' a sospechosos, obtuvieran previamente permiso del fiscal principal (ej.: el ministro de justicia ${ }^{70}$ ), proporcionando así un tipo de control supervisado de todo el proceso.

En cuarto lugar, el Estado puede poner en marcha campañas anticorrupción. Varios Estados post-comunistas - incluidos Bulgaria, la República Checa, Polonia (1994), Rusia y Eslovaquiahan puesto en marcha las llamadas campañas de 'M anos Limpias', emulando la campaña de manu pulite de principios de los noventa que acompañó a los escándalos de corrupción de Tangentopoli en Italia ${ }^{71}$. 0 tra campaña importante fue la puesta en marcha oficialmente por el gobierno Albano en junio de 1998. Tales campañas a menudo obtuvieron publicidad no sólo en la prensa sino también en los medios de comunicación electrónicos. Por ejemplo, en julio de 1992, el presidente Walesa propuso televisar los principales juicios en materia de corrupción como una forma para hacer que el público estuviera más concienciado con los problemas y con cómo el Estado estaba enfrentándose a ellos.

Las campañas anticorrupción tienen un efecto legitimador en el hecho de que normalmente sugieren que el Estado se toma en serio la lucha contra la corrupción. Pero si esas campañas no están bien dirigidas, no tienen ningún impacto y si tienen algún impacto éste es negativo. Su impacto desaparece a lo largo del tiempo si son demasiado prolongadas, ya que los ciudadanos se vuelven casi inmunes a ellas, y la campaña empieza a sonar a mera retórica. Pueden incluso ejercer una influencia negativa y si se quedan en meras declaraciones y no van acompañadas de actuaciones con un verdadero sentido. Esto se debe a que el Estado puede incrementar la concienciación pública sobre el problema de la corrupción, pero después frustrar las expectativas de que tales medidas están en realidad siendo implementadas para combatir la corrupción. Por último, el público se puede volver cínico si algunos actores políticos superiores parecen estar reduciendo la importancia de la corrupción mientras que otros están en campaña política en su contra. Al entonces primer ministro de Polo- nia, Waldemar Pawlak, se le acusó ampliamente a principios de 1995 de ser 'poco severo' en materia de corrupción policial cuando pareció inicialmente reticente a aceptar la dimisión del jefe de la policía nacional, Zenon Smolarek ${ }^{72}$. Aún peor resulta la situacion de aquellos líderes que han dirigido campañas contra la corrupción y que han establecido cuerpos para investigarla y luego han cerrado tales cuerpos o han cesado a los que estaban a la cabeza de los mismos porque se avergüenzan de esas agencias 0 de los hallazgos individuales. Un ejemplo clásico de esto se produjo cuando el presidente Yeltsin cesó a su principal encargado de la lucha anticorrupción, Yurii Boldyrev, cuando éste comenzó a hacer denuncias de corrupción entre los aliados más cercanos al presidente $^{73}$.

Un quinto método que se ha utilizado en algunas partes del mundo es el principio de rotación (o transferencia). D e acuerdo con este principio, a los empleados se les traslada regularmente de una oficina a otra, asumiendo que de esta forma serán menos capaces de desarrollar redes de clientela y otras prácticas corruptas que están típicamente relacionadas con cargos de larga duración en un puesto determinado. La investigación dirigida en el servicio público indio por Frank DE ZWART revela que este método no carece de problemas, y que puede en sí mismo generar nuevas formas de corrupción y clientelismo ${ }^{74}$. Sin embargo, los Estados post-comunistas podrían indirectamente aprender lecciones de las experiencias de otros ${ }^{75}$.

En sexto lugar, los Estados post-comunistas pueden crear agencias extranjeras que estén especialmente experimentadas en combatir la corrupción y el crimen organizado, y que disponen de las técnicas y del equipamiento más sofisticado del mundo para usar en esta lucha. Por ejemplo, varios Estados post-comunistas se han unido a la Interpol en los años noventa. Más aún, tanto Rusia como Polonia tiene ahora oficinas del FBI en sus capitales; éstas existen principalmente para combatir el crimen organizado, pero su presencia tiene necesariamente como consecuencia un impacto en la corrupción. El FBI también estableció una Academia Internacional para la Ejecución de la Ley en Budapest en 1996, donde se entrena a oficiales de policía de multitud de Estados del ECO inter alia a combatir el crimen organizado y la corrupción. La razón principal por la que EEUU tiene interés en este proyecto está en que aparentemente existe una creciente preocupación americana de que los países del ECO están involucrados en el contrabando internacional de drogas; este tipo de delito a menudo involucra a los empleados públicos, y de forma más evidente a la policía y a los empleados corruptos.

Relacionada con esta última cuestión está el hecho de que los Estados post-comunistas pueden mejorar el intercambio de información sobre criminalidad entre ellos. Esto ya ha comenzado. Un ejemplo reciente de ello es que los ministros del interior de la Federación Musulmana-C roata y de la República de Serbia en Bosnia firmaron un acuerdo en junio de 1998 para cooperar más de cerca en la lucha contra el crimen organizado; dados los lazos 
existentes a menudo entre el crimen organizado y la corrupción en los países del ECO, esto debería a su vez tener un impacto sobre la corrupción. Si bien, se han dado pasos en esta dirección, todavía se necesita hacer muchos más de lo que se ha hecho.

De acuerdo con Arnold H eIDEN HeIM ER, uno de los métodos más efectivos para reducir la corrupción es mejorar las condiciones de los empleados, y en especial sus salarios. Cita a Singapur como primer ejemplo de un país queha reducido dramáticamente su nivel de corrupción incrementando los salarios. En esta conexión, resulta de interés resaltar que Singapur es el único país asiático que aparece en el ranking de los diez países menos corruptos del 'índice de percepción de corrupción' (CPI) publicado por «nternational Transparency» ( ocupando la posición número 7 en el índice de 1998) ${ }^{76}$. Desgraciadamente, HEIDENHEIM ER también reconoce que las perspectivas económicas a corto plazo de los Estados post-comunistas determinan que este método en particular de hacer frente a la corrupción no resulta ser una opción realista en estos países ${ }^{77}$.

A más largo plazo, potencialmente uno de los métodos más eficaces para reducir la corrupción es educar a la gente, en especial a los jóvenes que estén en una edad impresionable, acerca de por qué la corrupción y otras formas de comportamiento no ético constituyen un problema en la sociedad. Si bien este método requiere de una puesta al día en muchas sociedades occidentales ya establecidas también, el vacío 'moral' en la Europa post-comunista que muchos observadores identificaron a comienzos de los noventa determina que exista en especial la necesidad de tal tipo de educación en los países del ECO. Aunque no se puede esperar que esta aproximación a largo plazo comience a cosechar beneficios hasta dentro de muchos años, incluso si se introdujera mañana, educar a los actuales empleados en ética por medio de cursos intensivos es aún otro método en la armeria anticorrupción. Algunos Estados post-comunistas ya han comenzado este camino. D e esta forma, Polonia introdujo clases de ética para sus agentes de aduana a finales de los años 90 como respuesta a niveles aparentemente altos de corrupción entre ellos ${ }^{78}$.

Todos los métodos elaborados hasta la fecha están formados por iniciativas de los anteriores (ej.: por parte del Estado); dada la lamentable situación de muchos aspectos de la sociedad civil en la actualidad en la mayoría de los países post-comunistas ${ }^{79}$, resulta adecuado que nos centremos en aproximaciones de arriba abajo. Pese a ello, la caída del poder comunista determina que la sociedad civil puede y debe jugar un papel cada vez más importante en el marco de los países del ECO, y puede hacerlo en el ámbito de la lucha contra la corrupción. D e acuerdo con T RANG, existen tres vías por medio las cuales la sociedad civil puede combatir esta forma de criminalidad: los medios de comunicación; grupos privados que dirigen e informan (whistleblow) y grupos privados que inician o ejercen presión para que haya una nueva legislación ${ }^{80}$. M ientras que estas dos últimas vías todavía se encuentran muy poco desarrolladas como agencias con su propia iniciativa en el mundo post-comunista (ver más abajo), en varios países los medios de comunicación han jugado un papel esencial durante los años noventa a la hora de mostrar la corrupción. En Polonia, por ejemplo, los periódicosy las revistas tales como Prawo I Zycie, ZycieWarzzawy y la más chispeante N ie ya han adquirido por sí mismas una reputación como especialistas en periodismo de investigación. El mayor escándalo policial en Poznan, al que hemos hecho referencia más arriba, estalló en marzo de 1994, principalmente como consecuencia de un artículo en primera plana en la Gaceta Wyborcza. Y a finales de 1996, el escándalo que afecto al consejo de privatización húngaro estalló con la publicación en septiembre de un artículo en el semanario económico Figyelo.

Sin embargo, desgraciadamente, los medios de comunicación se han visto silenciados a la fuerza en al gunos países post-comunistas cuando se ha percibido por parte de las autoridades estatales que se habían vuelto demasiado críticos con una corrupción sensible a la política. Junto con la bien conocida situación en la Eslovaquia de M eciar y en el Belarius de Lukashenka, se cerró el periódico ucraniano Política a finales de 1998, a juicio de su editor jefe por su publicación de una serie de artículos sobre la presunta corrupción en la administración del presidente Kuchma ${ }^{81}$.

Aunque la dirección de la corrupción iniciada por los ciudadanos está en su momento naciente en la mayor parte de los países de la región, la influencia del índice de 'Transparency International» crece rápidamente. Establecida tan recientemente como lo fue en 1993 como una organización internacional que perseguía eliminar 0 al menos reducir la corrupción grave en las principales transacciones de negocios internacionales (especialmente aquellas que requerían de fondos públicos), TI había establecido sucursales local es en seis países post-comunistas (Bulgaria, la República Checa, Hungría, Latvia, Polonia y Eslovaquia). Cuatro países más del ECO (Estonia, Rumania, Rusia y U crania) estaban en el proceso de establecer sucursales locales, mientras que un tercer grupo (Albania, Bosnia y H erzegovina, Croacia, G eorgia, Lituania, Eslovenia y Yugoslavia) disponía de contactos nacionales. Aunque se establecieron con gran influencia de la propiaTI, dichas sucursales locales estaban compuestas por nacionales de cada país correspondiente. Así, ésta es una forma a través de la cual los ciudadanos dentro de los países del ECO pueden aprender de la gente de fuera cómo luchar contra la corrupción, pero a la vez desarrollar con el tiempo sus propias infraestructuras, y métodos que resulten especial mente adecuados para sus propias necesidades y condiciones.

TI no es un cuerpo dirigido por el Estado ${ }^{82}$, y las agencias gubernamentales no pueden unirse a él. Para concluir esta visión general de los métodos que pueden utilizarse para combatir la corrupción, merece la pena pasar de una agencia que opera principalmente al nivel de la sociedad civil a considerar los papeles que pueden jugar las agencias internacionales patrocinadas oficialmente y las agencias supranacionales, a la hora de combatir la 
corrupción post-comunista, al igual que considerar el papel que pueden desempeñar aquellas empresas transnacionales.

El primer punto que debemos resaltar es que ha sido sólo recientemente cuando han empezado las agencias supranacionales a centrarse en la corrupción (en general y no exclusivamente en los países del ECO). Por ejemplo, cuando la OCDE dictó sus «Recomendaciones sobre el Soborno en las Transacciones C 0 merciales Internacionales» en mayo de 1994, estas directrices ge nerales se describieron como 'el primer acuerdo multilateral entre gobiernos para combatir el soborno a los empleados públicos extranjeros ${ }^{83}$. D esde entonces, la mayor parte de las principales organizaciones políticas y económicas internacional es y supranacionales se han concienciado acerca del problema de la corrupción y han adoptado varias declaraciones y directrices. Entre las más notables se encuentra la 'D eclaración contra la Corrupción y el Soborno en las Transacciones Comerciales Internacionales' de Naciones Unidas (diciembre de 1996); las directrices anticorrupción del Banco M undial de agosto de 1997; 'La Convención sobre la Lucha contra la Corrupción de los Funcionarios Públicos Extranjeros en las Transacciones Comerciales Internacionales' más reciente aun de la propia OCDE (firmada por los 29 miembros de la OCDE y cinco países no miembros en diciembre de 1997, siguiendo las recomendaciones adoptadas por el Consejo de la OCDE en mayo de $1997^{84}$ ); y la 'Convención de De recho Penal en materia de Corrupción' del Consejo de Europa (adoptada por el Comité de M inistros del Consejo en noviembre de 1998, y ratificada por los 21 Estados europeos (incluidos diez Estados post-comunistas) en enero de $1999^{85}$.

$M$ ientras que todas las directrices a las que hemos hecho referencia en el último párrafo pueden afectar a los países del ECO, pero no van específicamente dirigidas a estos países, junto con ello, el Consejo de Europa ha adoptado medidas diseñadas explícitamente para hacer frente a los problemas del crimen organizado y de la corrupción en los Estados europeos post-comunistas. En junio de 1996, junto con el programa «Phare» de la UE, estableció el proyecto 'O ctopus', en el que no menos de 16 países de la ECO accedieron a participar (Albania, Bulgaria, Croacia, la República Checa, Estonia, H ungría, Latvia, Lituania, la República de M acedonia, M oldova, Polonia, Rumanía, Rusia, Eslovaquia, Eslovenia y U crania). El proyecto duró dieciocho meses, e incluyó principalmentelaintroducción de medidas diseñadas para facilitar una mayor cooperación entre los países en su lucha mutua contra el crimen. Las medidas incluían la armonización de legislaciones, crecientes intercambios de informaciones, y el establecimiento de adecuados servicios de dirección. Se celebró una conferencia en Estrasburgo en diciembre de 1997 como conclusión al proyecto, en la que se recomendaron una serie de medidas específicas a determinados Estados post-comunistas ${ }^{86}$.

0 tra forma por medio de la cual las agencias externas pueden ejercer una influencia sobre los Estados post-comunistas es animándoles a involucrarse en el establecimiento de sus propias nor- mas para sus encargados. D e esta forma, cualquier gobierno del ECO debería ser capaz de producir su propio grupo de normas sobre los costes de la construcción, por ejemplo, las que a su vez deberían estar a libre disposición de las compañías que necesitan encargos para un proyecto determinado ${ }^{87}$. Si una determinada empresa constructora sugiriera la construcción de un bloque de oficinas o de un aeropuerto a un precio muy alejado de los parámetros oficiales - tanto si ese precio fuera muy alto como si fuera muy bajo- entonces los potenciales inversores estarían avisados de la anormalidad del encargo. Si los gobiernos post-comunistas no se encargan de establecer un escenario normativo, las agencias extranjeras pueden producir tales normas comparando las experiencias de los inversores extranjeros y dando publicidad a éstas.

La participación de 0 ccidente a la hora de contener la corrupción en los Estados post-comunistas no debería y de hecho no se limita a lo que podríamos calificar como medidas algo paternalistas (aunque de gran ayuda y solicitadas). Tal y como hemosmencionado en varios momentos más arriba, 0 ccidentetambién puede y debería jugar un papel a la hora de dar ejemplo y debería adoptar medidas que tengan consecuencias positivas para los países del ECO. Una de las medidas más significativas de este tipo adoptada recientemente ha sido la decisión de Suiza de requerir a los bancos que denuncien cualesquiera depósitos sospechosos y otras transacciones a las autoridades suizas; dicha ley entró en vigor el 1 de abril de 1998. H asta entonces, Suiza formaba parte de un puñado de países (que incluían a Chipre y Luxemburgo) cuyas prácticas bancarias opacas hacían que fuera bastante sencillo que las ganancias obtenidas de forma ilegal por parte de bandas criminales o de funcionarios públicos corruptos en el ECO ( y en otroslugares por supuesto) pudieran esconderse y almacenarse.

Un último tipo de actor internacional que puede desempeñar un papel importante en la reducción de la corrupción en los Estados post-comunistas (y en otros lugares) es la empresa transnacional. De esta forma, a finales de 1998, la empresa Unilever, mitad inglesa y mitad holandesa, se fue de Bulgaria en protesta porque se le exigía que pagara continuamente sobornos ${ }^{88}$. Si más compañías hicieran esto, ehicieran pública tanto su decisión como las razones de la misma, la necesidad de inversiones extranjeras animaría a la mayor parte de los gobiernos post-comunistas a adoptar una actitud mucho más enérgica en sus intentos por reducir la corrupción.

La lista hecha más arriba de medidas que cabe adoptar para hacer frente a la corrupción no es en absoluto una lista exhaustiva, pero sí pone de manifiesto que los gobiernos, la sociedad civil, y las agencias externas tienen todas un papel que desempeñar en la lucha contra la corrupción. El dinamismo del post-comunismo puede ahora tomarse en cuenta para decidir si resulta probable que la actual situación tienda a deteriorarse aún más, que se quede estacionada o que quizá mejore. M ientras que tal discu- 
sión debe ser necesariamente especulativa, se argumentará en muchos países que la situación es favorable.

A la hora de discutir los posibles escenarios futuros, la situación de la corrupción no puede desligarse de los desarrollos económicos y políticos más generales en determinados países. Por ejemplo, los continuos e importantes problemas económicos harán que resulte mucho más difícil reducir los niveles de corrupción. M ás aún, los pasos dados hacia la dictadura pueden posiblemente incrementar más que contener y reducir la corrupción. Este punto requiere de un mayor desarrollo.

Tal y como hemos indicado anteriormente, queda claro que muchos ciudadanos en el mundo post-comunista consideran que la razón principal que explica el aparente crecimiento en la corrupción en los años noventa está en el hecho de que los empleados públicos no temen las consecuencias de romper las reglas tanto como lo hacían durante la era 'totalitaria comunista'. Esto podría sugerir que los avances hacia otro tipo de autoritarismo - 0 un comunismo 'renacido', por ejemplo en Rusia - Ilevarían a reducidos niveles de corrupción. Pero esta afirmación debe cuestionarse, por tres razones.

En primer lugar, a medida que el poder se centraliza y se monopoliza cada vez más, las agencias potencialmente más poderosas para combatir la corrupción se debilitan. El papel de la socie dad civil, especialmente de los medios de comunicación libres, pierde importancia de forma marcada a medida que un país se aproxima a la dictadura. En segundo lugar - y no desligado del primer punto- antes dijimos que había un al to nivel de corrupción durante la era comunista (ej.: ino democrátical) en los países del ECO , si bien el público no estaba tan al tanto de la escala de esto como lo habría estado con la existencia de medios de comunicación más libres. Por último, una mirada por encima al índice CPI de IT revela que los países que parecen tener los niveles más bajos de corrupción son irresistiblemente Estados liberales de mocráticos, mientras que aquellos con los niveles más altos de corrupción son en su mayoría dictaduras, cuasi-dictaduras, países (tal y como Rusia) en los que el sistema bien funciona seriamente muy mal o esencialmente no funciona.

Por tanto, no resulta muy probable que los países post-comunistas que se apartan del camino de la democratización sufran al gún tipo de reducción en la corrupción, si es que experimentan alguna reducción; puede que el fenómeno no se comunique y discuta tan abiertamente en los medios de comunicación, pero esto no significa que haya desaparecido o se haya rechazado. En el momento actual, el país del ECO respecto del cual esta observación resulta más pertinente es Belarus. Pero Eslovaquia parece haber ido encaminada en esta dirección hasta finales de 1998 (la derrota de M eciar), mientras que la continua situación de intranquilidad en países tales como Albania y Rumania también causa preocupación. El caso de Rusia es, desde muchos puntos de vista, sui generis, pero no debemos pasar por alto la posibilidad de que surja una dictadura o cuasi-dictadura.
Al otro lado del espectro se encuentran los países post-comunistas que realmente parecen estar consolidando la democracia a finales de los noventa. Los países que se encuentran de forma más evidente en este caso son los cinco países que han recibido el sello de aprobación de la UE al ser nombrados como los primeros a la cabeza de la carrera para ser admitidos a la UE a principios del próximo milenio, que son la República Checa, Estonia, Hungría, Polonia y Eslovenia ${ }^{89}$. No nos preocupa aquí si son 0 no admitidos; sino más bien, la cuestión importante aquí es que observadores externos han identificado a estos países como países que ya han conseguido mucho en el proyecto de democratización y marketización. En todos los casos, salvo en el caso checo, el crecimiento económico ha sido positivo y bastante fuerte durante varios años ya, lo que supondría que, el argumento expuesto con anterioridad respecto a H EIDENHEIMER, en el sentido de que los Estados post-comunistas no podrían permitirse reducir la corrupción pagando a los empleados públicos salarios decentes, se volvería innecesario dentro de, quizá, una década ${ }^{90}$.

Tanto este grupo que está en primera línea para acceder a la UE, como aquel grupo de países que no ha tenido tanto éxito, pero que también parece no sufrir una situación de peligro inminente de convertirse en dictaduras o cuasi-dictaduras (tal y como es el caso de Bulgaria y, aunque discutible, también de Eslovaquia desde 1998), han avanzado no sólo en la esfera económica, sino también en la esfera política. Con esto queremos decir, por encima de todo, que el tipo de institucionalización de los procesos que Yufan ha visto en el contexto chino como necesario, y función esencial de la lucha contra la corrupción también es aplicable de igual forma a los países del ECO ${ }^{91}$.

\section{Conclusiones}

Se ha argumentado en este trabajo que la relación entre el neo-liberalismo y la corrupción en los Estados de la ECO es más compleja de lo que parece ser en los Estados occidentales (aunque estaúltima relación también garantiza una investigación más a fondo aunque no aquí). Se ha planteado la hipótesis de que el neo-liberalismo simultáneamente ha contribuido y ha limitado los aparentemente altos niveles de corrupción en los países del ECO. Por un lado, los Estados post-comunistas que han implementado menos reformas se perciben con carácter general como más corruptos que aquellos que han introducido mayor número de reformas. Por otro lado, la introducción paulatina de políticas neo-liberales genera nuevas oportunidades para la corrupción, y se ha argumentado que, si el contexto internacional lo hubiera permitido, el intento de tránsito del comunismo a la social democracia probablemente hubiera venido acompañado de niveles 
más bajos de corrupción. Dado que esa opción no estaba al alcance de ninguno de esos países, el neoliberalismo emerge como el menor de los dos males. En un mundo de relatividades, la corrupción con un buen crecimiento económico es mejor que la corrupción con poco o ningún crecimiento económico, ya que es más probable que la última situación agrave las tensiones sociales y políticas, en especial en el caso de los frágiles Estados de transición. M ás aún, incluso a la vez que contribuye a que haya niveles de corrupción relativamente altos, el racionalismo económico puede crear una dinámica que permitiría a los Estados del ECO reducir estos niveles en el futuro.

Antes de terminar este trabajo, quizá sea necesario analizar un aspecto no muy favorable - una pequeña deconstrucción - a la hora de analizar la corrupción post-comunista. Hasta este punto, ha habido una asunción implícita de que es bueno reducir la corrupción, e incluso mejor (si irrealista) erradicarla del todo. Desde una perspectiva ética, esa afirmación no es cuestionable. Desdeuna perspectiva coste efectividad, por otro lado, esa afirmación no resulta tan evidente por sí misma. Al menos desde que el análisis clásico Joseph N Y de la corrupción en términos de costes y beneficios, publicado hace más de tres décadas ${ }^{92}$, al gunos analistas han argumentado que la corrupción puede tener efectos positivos en el desarrollo de la economía (menos una forma de gobierno, aunque una economía que funcione bien puede subsiguientemente tener efectos positivos en el proceso de democratización por medio de una legitimación eudemónica ${ }^{93}$ ). Q uizás, la metáfora más utilizada en este contexto sea la de «lubricación» 0 «engrasamiento» de una máquina 0 una puerta.

M ás aún, al menos un observador, de los países del ECO y que sigue viviendo en los países del $\mathrm{EC} O$, ha argumentado que el 'problema' de la corrupción en los países del ECO es en parteuna función de la imposición de los valores occidentales. D e esta forma el profesor de D erecho András Sajó mantiene:

El hecho de que la forma en que el público entiende la corrupción se vea influenciado por las categorías occidentales, no significa que no exista corrupción en la Europa del Este, sino únicamente que la forma en la que se percibe tal corrupción no se debe siempre a genuinos factores endógenos. Si no fuera por el «toque de tambor» de la crítica externa, la corrupción no se entendería como un problema social grave, al menos no en la Europa Este Central ${ }^{94}$.

Además de mantener esto, el profesor Sajó explica este desarrollo sobre todo en lo relativo a la 'globalización de las comunicaciones y la información' que han hecho que 'sea virtualmente inevitable ${ }^{95}$ que se etiquete a estos países externamente'. Uno podría preguntarsesi SAjó puedeo debería aislar a ECO del resto del mundo - especialmente en el caso de países del ECO que han elegido ellos mismos hacer todo esfuerzo posible para ser admitidos en los 'clubes occidentales'- y también cabe pregun- tarsesi es o no legítimo pretender que existe una dimensión esencial e genuinamente endógena propia de los países del ECO (a la que reclama tener un acceso privilegiado ${ }^{96}$ ), más de lo que pue de haberlo en otros páses y culturas. Las culturas están viviendo fenómenos complejos y mutables, y su argumentación corre el riesgo de entenderse como reduccionista, estática y parroquial. M ás aún, no cabe ninguna duda de que una de las razones principales a las que se debe la percepción interna de altos niveles de corrupción en los Estados del ECO es el uso que los políticos, partidos políticos y medios de comunicación domésticos hacen de las alegaciones en materia de corrupción ${ }^{97}$. Pero sí que tiene algo de peso la noción de Sajó de construcción externa. Quizá, lo más importante es que nos recuerda (quizá de forma no intencionada) que 'la gente que vive en casas de cristal no debería tirar piedras'; los críticos occidentales de la corrupción en ECO debe rían reconocer queal gunos Estados occidentales están dando muy mal ejemplo. Al menos los Estados post-comunistas pueden ale gar la fragilidad e incertidumbre de la transición como razón que explica muchos de sus problemas.

0 tro argumento provocador $-y$ de nuevo estimulante- es el argumento que proviene de la propia ECO del Director del Centro de Estrategias Liberales en Sofia, I van K rastev. Éste argumenta que la corrupción puede parecer útil para aquellos gobiernos post-comunistas que no quieren enfrentarse directamente al problema de la falta de competitividad de sus economías. En bre ves palabras, argumenta que un cierto grado de tolerancia del gobierno hacia la corrupción constituye una forma de proteccionismo, y en este sentido incluso una forma de patriotismo. D ada esta situación, sugiere que los intentos por parte de las agencias occidentales externas (cita en particular al IM F y al Banco M undial) de combatir la corrupción post-comunista pueden ir mal encaminados, dado que, a la vez que urgen a los gobiernos de ECO que sean 'sensibles' frente al problema de la corrupción, a menudo ellos mismos son insensibles a los ben eficios potenciales de algunas formas de corrupción de los páses post-comunistas. M ás aún, apunta el hecho de que los populistas contrarios a la reforma tales como Lukashenka pueden utilizar la retórica anticorrupción en beneficio de su propio interés en que no haya ningún tipo de modernización ${ }^{98}$.

A un nivel, los argumentos tanto de Sajó y KRASTEV constituyen correctivos bienvenidos a asunciones demasiado repentinas y esencialmente incuestionables mantenidas por muchos observadores occidentales. Pero ambos, corren el peligro de debilitar el significado a largo plazo de la corrupción en sus sociedades. Tanto si nos centramos en la legitimación doméstica y sus implicaciones, como si nos centramos en la legitimación externa y sus ramificaciones, las percepciones obtenidas durante un periodo suficientemente prolongado de tiempo, relativas a que existe demasiada corrupción en una determinada sociedad, y que los gobiernos son demasiado tolerantes con esto, tiene un efecto negativo. 
En atención a la legitimación doméstica (si los gobiernos cambiantes con diferentes puntos de vista en el espectro democrático político parecen ser igualmente incapaces de hacer frente a los problemas), primero los regímenes, y después los sistemas, sufren, si existe una correlación en la mente pública entre la corrupción y los proyectos de democratización y marketización/ privatización en su conjunto. Esto puede abrir el camino al surgimiento de políticas antidemocráticas; y tal y como argumentábamos más arriba, esto no ayudará necesariamente a aliviar el problema de la corrupción, pero sí queintroduce numerosos problemas nuevos.

El problema de rechazar la legitimación externa en el contexto del fracaso de los gobiernos post-comunistas para tomar bajo control el problema de la corrupción está en que la inversión extranjera que resulta vital para la mayor parte de los países de ECO empieza a escasear 99; las implicaciones negativas de esto, incluidas lasimplicacionesnegativas sobreel sistema de legitimidad, son evidentes. A un nivel mucho más amplio, debemos resaltar queen losinformes delos individuosquesolicitan ser miembrosdelaUE, sólo un área de problemas en la sección de criterios políticos que se identificó respecto de cada uno de los países de ECO que solicitaban ser miembros fuela corrupción ${ }^{100}$. Si losEstadosdel ECO consideran que formar parte de la UE les ayudará a consolidar y a desarrollar sus proyectos de democratización, marketización y le gitimación, entonces - tanto si el problema de corrupción se ha exagerado o distorsionado por medio de las agencias occidentales- se les deberá ver tomándose la lucha contra la corrupción en serio. M uchos claramente ya lo han hecho. Por ejemplo, Latvia, habiendo sido fuertemente criticada por su corrupción, tanto por parte del Banco M undial como por la UE en los últimos años ${ }^{101}$, hasido alabadarecientemente por dar importantes pasos para hacer frente a la corrupción ${ }^{102}$. Bulgaria es otro país que se está tomando la lucha contra la corrupción en serio; su Coalición 2000, por ejemplo, está desempeñando un papel muy activo a la hora de tratar de reducir los actosilegales delos em pleados. Sin embargo, por las razones ya desarrolladas con anterioridad, demasiados miem- bros de las elites del ECO tienen un interés creado en que el Estado continúe tolerando niveles relativamente altos de corrupción, en línea con el concepto de 'equilibrio de reforma parcial' de HELLM AN. Se debe conseguir que se conciencien de que las acciones que dirigen en su propio interés no sólo hacen daño a otros dentro de sus propias sociedades, sino que a la larga tendrán un efecto de rebote sobre ellos mismos como extranjeros que se niegan a invertir en sus países ( inversión que constituye en último término la fuente principal de gran parte de sus riquezas) y que se nie gan a aceptar los sospechosos grandes depósitos que se realizan en bancos extranjeros.

Además, junto con esta última cuestión, está el hecho de que, a medida que pasa el tiempo, resulta más probable que más y más miembros de las elites post-comunistas aboguen por una legislación diseñada para legitimar la riqueza que han adquirido de forma cuestionable (por ejemplo, para que pueda ser heredada de forma legal). Esto, junto con el hecho de que la mayor parte de los Estados post-comunistas, aunque en diferente grado, están de forma gradual poniéndose al día en sus retrasos legislativos, sugiere, que aunque el cuadro de la corrupción es deprimente, no está carente de algún tipo de esperanza. Después de todo, al haber 13 de los 15 gobiernos de la UE que actualmente se han ido del centro, existe alguna esperanza de que se suavicen los salientes más puntiagudos del neo-liberalismo en los años venideros, con efectos que van más allá de una UE ampliada que incluye a algunos Estados del ECO. Dicho esto, no queda sitio para la complacencia. Incluso permitiendo la existencia de diferencias entre ellos, la todavía esencialmente frágil naturaleza de los Estados en transición del ECO supone que la corrupción constituye un problema mayor en estos páses que en aquellos páses que tienen democracias consolidadas y estables. Por razones que ahora deberían ya ser evidentes, el grado en que los Estados postcomunistas se tomen en serio la lucha contra la corrupción constituirá un indicador clave de la seriedad a la hora de consolidar no sólo el Estado de Derecho y la democracia, sino también la economía de mercado.
Artículo traducido por Cristina ALBA M uñ Oz.

* University of Melbourne.

1 La literatura existente en materia de corrupción es abundante. Aquellos lectores que deseen iniciar su trabajo de investigación con algunos de los trabajos comparadosmejores más recientes deberían ver A. H eID EN HeIM ER, M. JOHNSTON Y V. LeVIN E (eds.), Political Corruption (New Brunswick Transaction, 1989) D. D ELLA PORTA, Y. M ENY (eds.); D emocracy and Corruption in Europe (London Pinter, 1997) P. H EYwood (ed.), número dedicado a Political Studies, 45 (3), 1997 y J. N ewell y M. Bull (eds.), número especial dedicado a Law and Social Change, 27 (3-4). Para ver colecciones que se refieran de forma más concreta a la corrupción en los países postcomunistas de la CEE y la FSU, ver East European Constitutional Review, 6 (4), 1997, pp. 69-98 Transitions, 5 (3), 1998, pp. 16-67 y uno de los varios artículos sobre D emokratisatsiya 6 (4), 1998, pp. 613-91.
2 Ver Warsaw Voice, 22 de febrero de 19981997 (disponible en la web en la dirección http: www.warsawvoice.com.pl v453 indexhtml

3 Pese a habérsele declarado culpable por corrupción, en abril de 1994 y pese a haber sido sentenciado a doce años de prisión, N ano regresó de forma espectacular convirtiéndose de nuevo en primer ministro en 1997. Desgraciadamente para él, perdió de nuevo su cargo en 1998; una de las múltiples razones para ello fue casi sin ningún tipo de duda las fuertes críticas dirigidas a su persona por la OSCE, únicamente unos días antes de su dimisión, poniendo de manifiesto su fracaso a la hora de conseguir controlar la corrupción en Albania.

${ }^{4}$ M onitor (Fundación de Jamestown), 3 de mayo 1999 (on-line).

Para los detalles ver S. KeTTLE, «O f M oney and M orality», Transition, 1 (3), 1995, pp. 37- 9. Para ver comentarios más recientes relativos al proceso de privatización checa ver RFE/RL, 5-14 enero de 1999. 
${ }^{6}$ A este respecto ver Z. SZILAGYI, «Privatization Scandal T hreatens Coalition'sFuture and Public Confidence», Transition, 2 (25), 1996, pp.46-7 y 64. La privatización relacionada con los escándalos de corrupción continúa en Hungría; ver, por ejemplo, RFE-RL N ewsine, 11 de agosto de 1998.

7 Para ver detalles del escándalo policial Poznan el «Summary of World Broadcasts» de la BBC para Europa Central y O riental emitido entre las fechas de 12 de marzo de 1994, y 10 de febrero de 1995.

8 Transitions, 5 (5), 1998, p. 12.

${ }^{9}$ M onitor, 28 de diciembre de 1995 y 25 de enero de 1996.

${ }^{10}$ Ver, por ejemplo, P. Felgen GaUER, «V voennom vedomstve gotovitsya bol'shaya chistka», Segodnya, 6 de julio de 1996, p.1.

11 RFE/RL, 14 de noviembre de 1997, 17 de noviembre de 1997, 19 de diciembre de 1997.

12 Transitions, 5 (6), pp. 10-11.

${ }^{13}$ RFE/RL 'N ewsline', 4 de septiembre de 1998.

14 RFE/RL, D aily Report, 10 de diciembre de 1998.

15 Ver N. H ODGE, «On the Run», Transitions, 6 (3), 1999, pp. 8-9, y V. ZORYA, 'Lazarenko, aun sujeto a investigación, se retrata así mismo como un partido dañado', U krainian Weekly, 3 de enero de 1999 (on-line).

${ }_{16}$ East European Constitutional Review, 8 (3), 1999, pp.4-5. 0 tro ejemplo reciente es el del anterior primer ministro de la República Serbia en Bosnia, Gojko Klickovic, contra quien se dictó una orden de arresto en mayo de 1998 por usurpación de la propiedad y abuso de su cargo.

${ }^{17}$ La expresión meat-eating tiene su origen en el Informe de la Comisión Knapp de 1973 sobre la corrupción de la policía en Nueva York. Hace referencia a aquellos empleados que exigen sobornos u otros incentivos de carácter ilegal antes de llevar a cabo las funciones que tienen obligación de llevar acabo en cualquier caso. Los meat.eaters se distinguen de los grass eaters, en que los últimos aceptan pero no solicitan activamente sobornos y otros favores.

${ }^{18} \mathrm{RFE} / \mathrm{RL}, 3$ de febrero de 1998.

19 Para un resumen tabular de los índices de crimen en 15 Estados post-comunistas ver L. H OLM ES, «C rime, Corruption and Politics: International and Transnational Factors» en A. PRAVDA y J. ZieLonKA (eds.), D emocratic Consolidation in Eastern Europe: International and Transnational Factors (provisional mentey en camino 0 xford University Press), tabla 1.

${ }^{20}$ V. Lun EeV, «korruptsiya, uchtennaya i fakticheskaya», Gosudarstvo i pravo, no 8, 1996, p.87. Para ver un ejemplo reciente de una figura judicial superior (el Procurador General de la Federación rusa y el Fiscal Jefe M ilitar, Coronel General Yurii D emin) argumentando que la mayor parte de las alegaciones en materia de corrupción hechas por políticos no son más que travesuras y demagogia que alegaciones basadas en pruebas existentes, ver ERM OLIN, entrevistando a D enim, Izvestiya, 31 de julio de 1999.

${ }^{21}$ B. PAnEV, «Economic Reforms and Corruption» en N. Genov (ed.) Human D evelopment Report: Bulgaria 1997 (Sofia: United Nations D evelopment Program, 1997), p. 24.

${ }^{22} \mathrm{RFE} / \mathrm{RL}$, 24 de noviembre de 1997.

23 Para ver cifras de los niveles relativamente bajos de procesamientos y condenas por corrupción en EEUU 1993-96 ver S. G LIN KINA, «The 0 minous Landscape of Russian Corruption», Transitions, 5 (3), 1998, p. 22. D ebería señalarse que la definición de corrupción utilizada para recopilar estas estadísticas, tal y como se cita por G LIN KINA, sugiere fuertemente que los datos que se citan sobre los casos de procesamiento y condenas por corrupción, aunque modestos, vienen a exagerar el número de los mismos, tal y como entenderían la mayor parte de los analistas.

24 Por ejemplo, se registraron 250 casos de soborno solo en Polonia en 1992, lo que representó un 80\% del aumento de estos casos en 1991, ver A. SABBAT-SWID LICKA, «T he legacy of Poland's 'Solidarity' Governments», RFE/RL Daily Report, 27 de octubre de 1993. Además de la corrupción de las fuerzas de policía Poznan a la que hemos hecho referencia al final de 7, el mayor escándalo estuvo relacionado con la FOZ ( 'Foreign D ebt Service Fund', Fondo al servicio de la D euda Externa) que se estableció a finales de la era comunista (en febrero de 1989). Aunque fue abolido a finales de 1991, la corrupción objeto de investigación duró hasta principios de los noventa.

${ }^{25}$ Comunicado personal al autor por parte del M inisterio, Varsovia, 24 de febrero de 1997. Estas cifras tan bajas contradicen los datos proporcionados en «fair to
M iddling», Warsaw Voice, 25 de mayo de 1997. D e acuerdo con esta fuente, se dieron 36 casos de corrupción sólo contra agentes de policía que fueron juzgados ante tribunales polacos en 1996, tres cuartos de ellos (i.e c. 27) tuvieron como resultado condenas. De forma más general, de acuerdo con la misma fuente, 326 casos de empleados públicos que aceptaron sobornos fueron juzgados durante ese mismo años ante los tribunales polacos, con el resultado de 70 veredictos de culpabilidad (i.e. c. 21\%). La pura intuición sugiere a este autor que estas figuras más altas son o bien más precisas que las que le suministró el M inisterio, o que estas últimas se refieren sólo a aquellos casos que acabaron con encarcelamientos (espero clarificar este punto durante mi próxima visita a Varsovia). Aun así, estas cifras superiores son tambien muy bajas, en relación con las percepciones públicas de los niveles de corrupción.

${ }^{26}$ Panev, loc. cit., p. 24.

27 Oxford Analítica, East Europe D aily Report, 27 de septiembre de 1997.

${ }_{28}$ S. Kettle, loc cit., p. 39

${ }^{29}$ Los datos rusos (con la excepción de la última cifra) han sido amablemente proporcionados a este autor por Yurii Levada, Director of VTsIOM , en M oscú, 25 de abril de 1997. Lacifra de junio de 1998 esdeVT TIO M , M onitoring O bshchestvennogo M neniya, n. 3 ( mayo-junio), 1999, pp. 30-3.

${ }_{30}$ V. SHubkin y V. IVANova, «Strakhi na postsovetskom prostranstve: Rossiya, U kraina i Litva», M onitoring 0 bshchestvennogo M neniya, n.0 3 (mayo-junio), 1999, pp. 30-3.

${ }_{31}$ El informe de 1991, dirigido por CBOS, se recoge en RFE/RL Daily Report, 8 de agosto de 1991. los datos de 1995 han sido proporcionados amablemente por el profesor András Sajó durante una entrevista en Budapest, 24 de septiembre de 1996. 32 J. Mac, A. Niewrzedowski y Z. Stachura, W prost, 22 de junio de 1997, tal y como se informó en «The Right to Corruption», Warsaw Voice, 29 de junio de 1997.

33 Transparency International, Report of the M isión of Transparency International to the Slovak Republic and Rumania 20-29 mayo de 1996 (Berlin Transparency International, 1996), p.9

${ }_{34}$ PAN Ev, loc. cit., p. 24. D eberíamos resaltar que la lista de opciones estaba relacionada con la propiedad en su mayor parte, y no relacionada con todos los aspectos de problemas sociales, políticos y económicos, aunque una de las opciones fue 'el uso de narcóticos'. Para una descripción general de investigación longitudinal sobre las actitudes ante la corrupción en Bulgaria, que claramente apoya el argumento que hago aquí, ver «the Center for the Study of D emocracy's», M onitor (Sofia), I ssue 3, 1998, pp. 10-12.

${ }_{35}$ S. GIRNIUS en OM RI Daily Digest, 3 de octubre de 1995.

${ }^{36}$ Shubkin e Ivan ova, loc. cit., pp. 32-6. Sin que resulte sorprendente para aque Ilos familiarizados con la situación del crimen en los Estados post-soviéticos del Báltico y eslavos, los porcentajes actuales que optan por estas categorías de 'miedo' son mucho más altos en U crania que en Lituania.

${ }^{37}$ P. Zvagulis yJ. Cleave, «C orrupction Remains Widespread in Latvia», RFE/RL D aily Report, 15 de diciembre de 1998. Para encontrar pruebas de que, a mediados de los años noventa, el $75 \%$ de los lituanos consideraban que el «crimen (y, por tanto, no sólo la corrupción) era el segundo mayor problema en su país después del estado de la economía»; ver R. KRICKUS, «D emocratization in Lituania» en K. D awish a y B. PARROTt (eds.), The consolidation of democracy in East-Central Europe (Cambridge: CambridgeUniversity Press, 1997), p. 312, desgraciadamente, KRICKUS no nos proporciona ninguno tipo de detalles sobre esta fuente.

${ }^{38} \mathrm{~N}$. LuBIN, Central AsiansTake Stock (Washington DC: United States Institute of Peace, 1995), tal y como se recogió en Trends in Organised Crime, 1 (4), 1996, p. 84. Actualmentedirige informes en cuatro Estados post-comunistas (Bulgaria, H ungría, Polonia, y Rusia), diseñados para proporcionar pruebas más precisas sobre hasta que putno los ciudadanos asocian la corrupción con los proyectos de democratización y marketización.

${ }^{39}$ W. Miller, A. Grodeland y T. Koshech Kina, «Are the people victims or accomplices? The use of presents and bribes to influence officials in Eastern Europe?», Crime, Law and Social Change, 29 (4), 1998, pp. 273-310.

${ }_{40}$ S. GIRnIUS, loc, cit.

${ }^{41}$ Podemos encontrar los índices CPI en la página web de 'Transparency'www.transparency.de. Desgraciadamente, el índice CPI de 1991 no estaba todavía disponible en el momento en que escribo este trabajo. 
42 En el momento en el que escribía este trabajo, no había podido obtener el libro de 7.000 páginas de Freedom House, al que se refiere este punto y, consecuentemente, no había podido estudiar la metodología utilizada para producir este orden por ranking. Espero poder analizar la metodología de 'Freedom House', y poder compararla con la de TI, antes de que este trabajo se publique. La fuente de información aquí incluida es Turknews, 15 de diciembre de 1998.

${ }^{43} \mathrm{El}$ uso de 'el camino de la dependencia' aquí se da desde una perspectiva en la que existen limitaciones en las estructuras, instituciones y recursos; no debería interpetarse como sinónimo del determinismo cultural. En esto, la posición está a la par de la que yo entiendo que es la postura de D avid STARK; ver D. STARK, «Path D ependence and Privatization Strategies in East Central Europe», en R. SCHönfeld (ed.), Tranformation der Wirtschaftssyteme in Ostmitteleuropa (M unich: SüdosteuropaGesellschaft, 1993), pp. 11-39. Para una muy sucinta pero útil introducción a los conceptos de «el camino de la dependencia» en el contexto del post-comunismo ver K. HENDERSON y N . ROBINSON, Post-Communism Politics (London: Prentice Hall, 1997), pp. 30-1 y 39 .

${ }^{44}$ Ver, por ejemplo, J.C. Sc0тt, Comparative Political Corruption (Englewood Cliffs: Prentice H all, 1972), pp. 16, 77 y 87. Siendo justos con ScotT, existía poca evidencia sobre la existencia de corrupción en los Estados comunistas en el momento de escribir este trabajo.

${ }^{45}$ Para un análisis comparado ver L. H oLm ES, The End of Communist Power (N ew York. 0 xford University Press, 1993).

${ }^{46}$ Citado en L. ZangA, 'Corruption Takes its Toll on the Berisha Government', Transition, 1 (7), 1995, p. 13.

${ }^{47}$ Incluso en Rusia, donde un porcentaje bastante más alto de empleados son formalmente acusados de corrupción de lo que lo son en muchos Estados post-comunistas, el número de casos de corrupción oficial registrados entre mediados de los ochenta y mediados de los noventa fue once veces mayor que el número de conde nas reales; ver LuneEv, loc. cit., p. 86.

${ }^{48}$ Desgraciadamente, la cuestión del crimen organizado se escapa del ámbito del presente artículo, al igual que la pregunta directa más relevante relativa a las conexiones entre el crimen organizado y la corrupción. Los lectores interesados en estos dos temas podrían comenzar su búsqueda de fuentes literarias con T. FRISBY, "T he Rise of O rganised Crime in Russia: Its Rotos and Social Significance», Europe Asia Studies, 50 (1), 1998, pp. 27-49 y las cuestiones planteadas en el East European Consitutional Review y Transitions citado in fine 1. Para ver análisis más populares, ver, por ejemplo, C. SterLING, CrimeW ithout Frontiers (London: Warner, 1995) y B. Freem antLe, The 0 ctopus (London: O rion, 1995).

49 Para ver un argumento explícito en el sentido de que la forma de reducir la corrupción es reducir el papel del Estado en la economía, ver V. T ANzI, «Corruption, Governmental Activities, and M arkets», Finance and Development, 32 (4), 1995, pp. 24-6. En contraste con la posición defendida por T anzI, se argumenta aquí que no sólo importa el nivel de intervención del Estado sino la naturaleza de dicha intervención. El argumento de T ANZI, sólo tiene sentido si el Estado no interviene en absoluto en la economía, sino que se encarga del outsourcing y continúa supervisando las funciones que con anterioridad él desempeñaba. Si la forma «pura» de Estado, un Estado no intervencionista identificada por T AnzI, fuera a implementarse, podrían tener como resultado que numerosos bienes colectivos no se proporcionaran en absoluto.

${ }_{50}$ Este punto nos ayuda a explicar los problemas políticos relacionados con las leyes de conflicto de intereses, a las que nos referimos en la tercera sección de este trabajo.

${ }^{51}$ Cabría aducir que la triste actuación económica de Rusia en los últimos tiempos podría disuadir a otros páses post-comunistas del neo-liberalismo. Pero ese sería un argumento erróneo. Una de las principales razones que explican los problemas que ha tenido Rusia está en el hecho de que no siguió hasta el final con sus reformas. Se paró en dichas reformas en algún lugar entre la dirección de la economía y la economía de un mercado radical, principalmente debido a disputas políticas domésticas acerca de cuál era la mejor manera hacia delante ( 0 hacia atrás) y debido a las excentricidades de un presidente enfermo. Sufre de un caso grave de coitus 'reformus economicus' reseratus (mis disculpas a los estudiosos del latín). Esta situación confusa ha exacerbado el problema de la corrupción.

52 De acuerdo con el Banco Europeo de Reconstrucción y D esarrollo, para 1999 solo tres de los países post-comunistas (Polonia, Eslovaquia y Eslovenia) habían sobrepasado sus propios niveles de GDP de 1989, mientras que H ungría acaba de alcanzarlos; ver The Economist, 351 (8116), 1999, p. 112.

${ }^{53}$ A. Giddens, The Third Way (Cambridge: Polity, 1998)

${ }^{54} \mathrm{D}$ e acuerdo con el trabajo producido por IM F en 1998 por Jeffrey Sachs, incluso aquellas proyecciones de crecimiento relativamente optimistas para los países de Europa Central, sugieren que estos países tardaran al menos décadas en ponerse al día con los Estados más pobres de la EU, como $G$ recia. Para ver un resumen breve y un histograma ver Business Central Europe, 5 (56), 1998, p. 64

${ }_{55}$ M. T ARM (artículo sin nombre), City Paper- Blatic States, reproducido en Eastern Europe Business N etwrk (on-line), 19 de agosto de 1996.

${ }^{56}$ El caso de Latvia también plantea dudas acerca de la hipótesis de que el tamaño constituye un buen indicador de los niveles de corrupción. De acuerdo con este argumento, los países más pequeños tienden a ser menos corruptos que los países más grandes. De hecho, sí que parece existir al gún tipo de relación estrecha razonable entre el tamaño de la población y su ranking en el índice CPI de IT. Sin embargo, el tipo de sistema político y el nivel de desar rollo económico tambien parecen ser partes de esa ecuación, así que debemos preocuparnos respecto de aquellas correlaciones demasiado simplificadas y reduccionistas bivariadas (por tanto, de este tipo de explicaciones).

${ }^{57}$ Por tanto, la deuda externa de Polonia se redujó dramáticamente de un día para otro a principios de los años noventa. Existe sin duda al go de ironía en el hecho de que tantos políticos occidentales de derechas y banqueros hayan tratado a un líder sindical con tanto respeto, incluso con admiración. Sin embargo, también resulta irónico que Walesa a menudo estuviera de parte de los neo-liberales tales como Balcerowicz y Suchocka en contra de sus anteriores colegas de Solidaridad.

${ }^{58}$ J. H elLm AN, «W inners TakeAll: The Politics of Partial Reform in Postcommunists Transitionss, World Politics, 50 (2), 1998, pp. 203-34, y aquí en especial pp. 204 y 233.

${ }^{59} \mathrm{He}$ argumentado en otros sitios que el aparente incremento en la corrupción occidental en los últimos años tambien se debe en parte a la incompleta aplicación de los principios neo-liberales en el mundo real de las economías y de la política; ver L. H OLm ES, «C orruption in Europe», Dialogue, 18 (2), 1999, pp. 19-25. Sin embargo, resultaría bastante incorrecto deducir de esto que soy una defensora de un 'puro' y radical neo-liberalismo. Más bien mi argumento es que la implementación de una ideología en su fórmula pura ocasionaría menos problemas de los que se están manifestando en la actual implementación de políticas basadas en el neoliberalismo, pero que resulta peligroso defender ideologías radicales que nunca pueden llegar a ponerse en práctica de un modo adecuado. Criticar a los humanos por ser imperfectos e irracionales, y creer que se pueden superar tales imperfecciones, es donde radica la raíz del totalitarismo; uno tiene sólo que pensar en los conceptos de Ü bermensch o de 'la nueva persona socialista' para que esto resulte evidente.

${ }^{60}$ Esta sub-sección depende en su mayor parte de un análisis sobre los métodos para combatir la corrupción post-comunista I publicado en 1997, aunque esta versión esta actualizada y truncada en comparación con la original; ver L. H OLMES, «Corruption in Post-Communist Countries, with Particular Reference to Poland» en L. H o LM Es y W. Ro szkow SkI (eds.), Changing Rules (Warsaw: ISP PAN, 1997), esp. pp. 157-65.

${ }^{61} \mathrm{M}$ erece la pena resaltar aquí que un veterano actor político checo ha argumentado recientemente de su propio país que «... los políticos se dan ahora cuenta de que la palabra clave ya no es 'economía de mercado', sino 'Estado de D erecho'»; ver J. PeHE, «T he D isappointments of Democracy», Transitions, 5 (5), 1998, p. 42. Si la observación de PeHE es correcta, ésta es una señal que nos anima a combatir contra la corrupción, que a la vez ayudará tanto a los proyectos de democratización como a los de marketización.

62 Sin embargo, éste no es siempre el caso. D e esta forma, el anterior ministro de Armeniadela Industria Eléctrica Rudolf Teymurazian fuesentenciado a ocho años de prisión y se le ordenó que pagará aproximadamente 500.000 dólares americanos de multas en septiembre de 1998 por desfalco. En cuanto a las sumas de dineros involucradas, se impuso una condena aún más dura al parlamentario lituano y anterior ministro de defensa AndriusButkeviciusen 1998, quefuecondenado a cinco años y medio de prisión por aceptar un soborno de 15.000 dólares americanos.

63 "Fair to Middling», Warsaw Voice, 25 de mayo de 1997; presumiblemente, aquellos prisioneros que se comportaban adecuadamente podían solicitar la libertad bajo 
fianza en unos meses. Se recuerda al lector aquí sobre los problemas de la corrupción polaca elaborados in fine 25. Este problema con la imposición de condenas leves no es propio de los Estados post-comunistas, por supuesto; es una cuestión suficientemente conocida en 0 ccidente (se le mencionan de nuevo al lector los datos norteamericanos citados en G LINKINA, loc. cit., p. 22). Un ejemplo claro de esto se encuentra en el reciente caso de alto perfil. En diciembre de 1998, se declaró culpable por parte de un tribunal belga de corrupción al hombre que había dirigido la OTAN hasta su dimisión en 1995, W illi Claes, durante los años ochenta, cuando fue ministro de asuntos económicos de Bélgica. Pese a (o quizá debido a ) su alto cargo, se le trato con poca severidad, y obtuvo una condena que se suspendió de tres años de carcel, una multa de aproximadamente 1.600 dólares americanos, y la prohibición de ocupar un cargo público durante un periodo de cinco años. Esto apenas nos da un mensaje apropiado sobre si los gobiernos y los sistemas judiciales se toman en serio la política de supresión de la corrupción. Si O ccidente se toma en serio la reducción de la corrupción globalmente, podría y debería mandar mensajes menos ambi guos a otras partes del mundo sobre sus propias actitudes hacia la corrupción, como se ve en sus propias actuaciones.

${ }^{64}$ K. N IKLEWICZ, entrevistando a L. Kacperski, «Staring D own the Economic Mafia», Warsaw Voice, 5 de octubre de1997.

65 M ientras que aparentemente resulta bastante probable que la UE introduzca normas más duras para asegurar que los Euro-parlamentarios declaran todos sus intereses financieros externos (ver el European Voice, 7-13 enero de 1999, p.7), el gobierno liberal (conservador) australiano trata en este momento (1999) de hacer frente al problema del conflicto de intereses legalizando completamente la situación en la que los ministros tienen acciones de compañías que pujan por contratos estatales. Con anterioridad esto habría constituido un caso claro de 'conflicto de intere ses' en la mayor parte de las democracias occidentales. Esta aproximación, que el gobierno H oward alaba como una victoria del 'sentido común', es a la vez un reflejo del fuerte aumento en las ofertas que resulta de las políticas neo-liberales, y un reflejo del marcado declive de la ética del sector público en Australia. M ás que tratar de hacer frente al problema del conflicto de interés, el gobierno australiano trata de hacerlo desaparecer descriminalizando el comportamiento inadecuado. Sólo cabe deducir cuál es el mensaje que se está dando. Sobre la corrupción y la ética del sector público en Australia ver por ejemplo, G. CLARK, E. PrIOR JONSON y W. CaLdow (eds.), A ccountability and Corruption (St. Leonards, N SW: Allen and Unwin, 1997); R. TIffen, Scandals: M edia, Politics and Corruption in Contemporary Australia (Sydney: UN SW Press, 1999).

66 K. O KO LICSAnYI, «H ungarian Bank Closures: Regulators M iss the M ark», RFE/ RL D aily Report, 8 de septiembre de 1992.

67 RFE/RL, 5 de marzo de 1999.

68 M Ac et al., loc. cit, han defendido en el contexto polaco que 'M uchos políticos de la oposición, sin embargo, consideran que la verdadera «ley de corrupción» fuela ley que aprobaron los partidos políticos esta primavera (1999-LTH ). D eacuerdo con esta ley, los partidos políticos pueden financiarsetanto con el presupuesto nacional como por negocios, lo que supone que, por un lado, pueden representar intereses públicos $y$, por otro lado, pueden ser guardianes de confianza de los intereses negociales de aquellos quelesfinancian'. Por supuesto, estep roblema sobrelos potenciales conflictos de intereses de los partidos políticos es apenas desconocido en 0 ccidente.

${ }^{69}$ Tanto la República C heca como H ungría están también considerando propuestas para atrapar a los culpables, para hacer frentea la corrupción y al crimen organizado.

70 RFE/RL Daily Report, 11 de febrero de 1993 y 8 de junio de 1994.

71 Para un análisis sofisticado de la situación de la corrupción italiana y sus implicaciones políticas ver D. D ella Porta y A. Van nu cCl, «T he "PerverseEffects" of Political Corruption», Political Studies, 45 (3), 1997, pp. 516-38.

72 L. VINTON, «Polish Government under Siege», O mri Daily Digest, 31 de enero de 1995.

73 Se hizo de nuevo referencia a esto en los medios de comunicación norteamericanos; ver S. Sch m em an n M, «W hat M akes N ations Turn Corrupt?», N ew York Times, 28 de agosto de 1999 (on-line). Para un análisis de la lucha contra la corrupción de Boldyrev ver B. W HIT M ORE, «Russia's Top Crime Fighter», Transitions, 5 (3), 1998, pp. 35-9.

74 F. DE ZwarT, «Personnel Transfer in India State Bureacracy: Corruption and Anti-corruption» en H. BAKKer y N. SChulte-N ORdholt (eds.), Corruption and Legitimacy (Amsterdam: SISW 0, 1996), pp. 53-64.
75 Una de las aproximaciones más inusuales utilizada en otra parte del mundo - y a la que no haremos mención aquí- es la adoptada por las autoridades de la ciudad de M éjico para hacer frente al problema de la corrupción entre su policía de tráfico. $\mathrm{H}$ abiendo decidido que las mujeres son menos corruptibles que los hombres, jtransfirieron la tarea de poner multas de tráfico del cuerpo de policías de hombres al cuerpo de policías de mujeres! Ver Sunday Age, 1 de agosto de 1999, p. 12.

76 Singapur ha estado entre los diez primeros de forma constante (es decir, entre los diez países que se han percibido como menos corruptos) desde que el TI emitió su primer índice anual CPI en 1995.

77 A. HeId en helm ER, «ntroduction»en D. Trang (ed.), Corruption and D emocracy (Budapest: Institute for Constitutional and Legislative Policy, 1994), p. 18.

78 K. N IKLEWICZ, «Awash in Bribes», Warsaw Voice, 21 de septiembre de 1997.

79 Ver C. PICKVAN CE, «D emocratisation and the D ecline of Social M ovements: The Effects of Regime Change on Collective Actino in Eastern Europe, Southern Europe and Latin America», Sociology, 33 (2), 1999, pp. 353-72.

80 D. Trang, «Preface» en Trang, op. cit., p. 9.

81 RFE/RI Daily Report, 10 de diciembre de 1998.

${ }_{82}$ Para ver detalles sobre la propia obtención de fondos de TI (que en su mayor proporción proviene del proyecto de fondos, para el que la TI compite) ver la sección del extracto financiero en la página web de TI.

83 Transparency International, Sharpening the Responses against Global Corruption (Berlin: Transparency International, 1996), p. 15. Una parte revisada de estas recomendaciones fue aprobada por el Consejo de la O CDE en mayo de 1997.

84 Las directrices de la O CDE se han endurecido progresivamente, detal forma que para febrero 1999 se habían convertido en lo que The Economist (35 \&102>, 1999, p. 17) llamó en la primera norma internacional que convierte la realización de sobornos por parte de empleados públicos extranjeros en un delito.

${ }^{85}$ Los diez eran Albania, Bulgaria, Georgia, Latvia, Lituania, Polonia, Rumanía, Rusia, Eslovaquia, y Ucrania. Para mediados de agosto de 1999, otros tres Estados post-comunistas habían ratificado la Convención - H ungría (abril de 1999), M oldova (junio de 1999) y la república de M acedonia (julio de 1999).

${ }^{86} 0$ tro ejemplo de una agencia occidental que adapta y adopta una política anticorrupción a la CEE y a los países de FSU es la adoptada por USAID en diciembre de 1997; ver K. HENDERSON, «Corruption: W hat Can Be Done About It?», Demokratisatsiya, 6 (4), 1998, pp. 681-91.

87 Ésta parece ser una propuesta similar a la llevada a cabo en la reunión con fondos de Soros de expertos anti-corrupción en Nueva York en enero de 1998, donde hubo una discusión sobre «un proyecto de obtención pública». Bajo esta propuesta, «se recogerían y diseminarían datos, mostrando la variación en el coste de productos similares y servicios similares en jurisdicciones diferentes» [M. KaUfman, «C orruption: W hat Can People of Good W ill D o?», Transitions, 5 (3), 1998, p. 3]. 88 «A global war against bribery», Economist, 350 (8102), 1999, p. 23.

${ }^{89}$ Se recuerda al lector que todos estos países actuaron bien, en comparación con otros Estados post-comunistas, tanto en el índice CPI del TI (aunque la tabla de 1998 no se refiere a Eslovenia) como en el ranking de corrupción de Freedom H ouse. 90 Esteargumento puede parecer estar en contradicción con los expresadosen lasnotas depágina 52 y 54 . Sin embargo hay dos razones por las que esto no es así. En primer lugar, las estimaciones Ilevadas a cabo en la nota de pie de página 54 pueden resultar ser incorrectas (demasiado pesimistas). En segundo lugar, y con mayor relevancia, el argumento expuesto aquí se refiere a que determinados países de la EC 0 pueden ser capaces depermitirsesalariosmejores para sus empleadosen relación con lossalariosque obtienen otrostrabajadores dentro de esos mi smos países, y no en relación con los salarios obtenidos en otros países. Como economía en transición a punto de completarse y teniendo el Estado que pagar proporcionalmente menos en desarrollar o renovar las infraestructuras, debería haber más para pagar a los empleados.

91 Yufan $\mathrm{H}_{\mathrm{AO}}$, «nstitution-Building: An Unintended Consequence of Corruption in China in the 1990ss, trabajo no publicado presentado en el Congreso Internacional sobre Estudios Comunistas y Post-Comunistas, M elburne, julio de 1998.

${ }^{92} \mathrm{~J} . \mathrm{N}$ YE, «C orruption and Political Development: A Cost-Benefit Analysis», American Political Science Review, 61 (2), 1967, pp. 417-27. Para ver los argumentos de otros defendiendo ya a comienzos de los años sesenta que la corrupción era beneficiosa, especialmente en las primera etapas del despegue económico, ver, por ejemplo, Nathaniel LefF, «Economic Development Through Bureaucratic Corruption», The American Behavioural Scientist, 8 (4), 1964, pp. 8-14 y S. 
H UNTINGTON, «M odernization and Corruption» en su Political Order in Changing Societies (N ew H aven: Yale University Press, 1968), pp. 59-71. Estos tres trabajos, junto con otros que tratan este tema, pueden encontrarse en HEIDENHEIMER, JOHNSTON Y LE VINE, op. cit.

${ }_{93}$ Paraver un reciente «análisis costes-beneficios» de la corrupción en los Estados postcomuni stas ver L. H OLM ES, «DieV or- und N achteile der Korruption und dessen Reportage in Zentral. Und 0 steuropa» en Jahrburch für Europa- und NordamerikaStudien 3: Politische Korruption (Gottingen; ZEN S, en camino), 27 pp. En ms. Uno de los artículos más citados y más persuasivo que demuestra empíricamente que la corrupción tiene un efecto negativo en la inversión y el crecimiento económico es el de P. M AURO, «C orruption and Growth», Q uarterly Journal of Economics, 110 (3), 1995, pp. 681-712.

${ }_{94} \mathrm{~A}$. SAJÓ, «Corruption, Clientelism and the Future of the Constitutional State in Eastern Europe», East European Constitutional Review, 7 (2), 1998, p. 37.

${ }_{95} \mathrm{lbid}$, p. 38.

${ }_{96}$ M erece la pena señalar aquí que un estudioso polaco, que vive ahora en EEUU, ha escrito lo siguiente, «...trabajar en EEUU me dio la distancia que necesitaba para estudiar muchos aspectos de la realidad polaca de los que no había sido consciente mientras que vivía allís; ver J. KUBIK, The Power of Symbold Against the Symbols of Power (University Park, PA: Pennsylvania State University Press, 1994), p. 15. Soy totalmente consciente de que el profesor SAjó ha viajado mucho y ha trabajado en
O ccidente. También respeto absolutamente su derecho a hablar en defensa de su propio pás, criticando a 0 ccidente, e interpretando la corrupción desde una perspectiva radicalmente diferente de la mayoría de los observadores. Mi única preocupación es la de que él sea consciente de que a lo mejor está desechando aspectos importantes al criticar a 0 ccidente.

${ }_{97}$ Tampoco debe olvidarse para los que teman lo contrario quee índice CPI de TI revela que los occidentales no tratan a todos los países de la EC 0 igual, y que se dan cuenta que muchos de ellos son mucho menos corruptos que Rusia!

${ }_{98}$ I. KRASTEV, «D ancing with Anticorruption», East European Constitutional Review, 7 (3), 1998, pp. 56-8.

${ }_{99}$ Para ver un informe comparado que prueba que esto ocurre, ver (sin firmar), «Reducing Corruption», World Bank Policy and Reserach Bulletin, 8 (3), 1997, esp. p. 2.

100 Ver, por ejemplo, European Comisión, «Commission Opinión on Hungary's Application for membership of the European Union», Bulletin of the European U nionSupplement 6 (Luxemburgo: 1997), p. 20; European Commission, «C ommission 0 pinion on Slovenia's Appplication for Membership of the European Union», Bulletin of the European Union- Supplement 15 (Luxemburgo: 1997), p. 20.

101 P. Zvagulis y J. Cleave, «C orruption Remains W idespread in Latvia», RFE/RL $D$ aily Report, 15 de diciembre de 1998.

102 Ibid., RFE/RL D aily Report, 2 de febrero de 1998; D. SILVA, «Latvia named top corruption fighter», Baltic Times, 8-14 de abril de 1999. 